\title{
Detecting Biosignatures in the Atmospheres of Gas Dwarf Planets with the James Webb Space Telescope
}

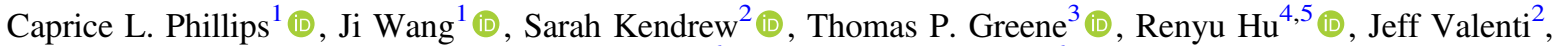 \\ Wendy R. Panero ${ }^{6}$ (D), and Joseph Schulze ${ }^{6}$ (iD \\ ${ }^{1}$ Department of Astronomy, The Ohio State University, 100 West 18 th Avenue, Columbus, OH 43210 USA \\ ${ }^{2}$ European Space Agency, Space Telescope Science Institute, 3700 San Martin Drive, Baltimore MD 21218, USA \\ ${ }^{3}$ Space Science and Astrobiology Division, NASA Ames Research Center, MS 245-6, Moffett Field, CA 94035, USA \\ ${ }^{4}$ Jet Propulsion Laboratory, California Institute of Technology, Pasadena, CA 91109, USA \\ ${ }^{5}$ Division of Geological and Planetary Sciences, California Institute of Technology, Pasadena, CA 91125, USA \\ ${ }^{6}$ School of Earth Sciences, The Ohio State University, 125 South Oval Mall, Columbus, OH, 43210, USA \\ Received 2021 May 27; revised 2021 September 18; accepted 2021 September 22; published 2021 December 16
}

\begin{abstract}
Exoplanets with radii between those of Earth and Neptune have stronger surface gravity than Earth, and can retain a sizable hydrogen-dominated atmosphere. In contrast to gas giant planets, we call these planets gas dwarf planets. The James Webb Space Telescope (JWST) will offer unprecedented insight into these planets. Here, we investigate the detectability of ammonia $\left(\mathrm{NH}_{3}\right.$, a potential biosignature) in the atmospheres of seven temperate gas dwarf planets using various JWST instruments. We use petitRadTRANS and PandExo to model planet atmospheres and simulate JWST observations under different scenarios by varying cloud conditions, mean molecular weights (MMWs), and $\mathrm{NH}_{3}$ mixing ratios. A metric is defined to quantify detection significance and provide a ranked list for JWST observations in search of biosignatures in gas dwarf planets. It is very challenging to search for the $10.3-10.8 \mu \mathrm{m} \mathrm{NH}_{3}$ feature using eclipse spectroscopy with the Mid-Infrared Instrument (MIRI) in the presence of photon and a systemic noise floor of $12.6 \mathrm{ppm}$ for 10 eclipses. NIRISS, NIRSpec, and MIRI are feasible for transmission spectroscopy to detect $\mathrm{NH}_{3}$ features from 1.5-6.1 $\mu$ m under optimal conditions such as a clear atmosphere and low MMWs for a number of gas dwarf planets. We provide examples of retrieval analyses to further support the detection metric that we use. Our study shows that searching for potential biosignatures such as $\mathrm{NH}_{3}$ is feasible with a reasonable investment of JWST time for gas dwarf planets given optimal atmospheric conditions.
\end{abstract}

Unified Astronomy Thesaurus concepts: Biosignatures (2018); Astrobiology (74); Exoplanet atmospheres (487); Exoplanet atmospheric composition (2021); Planetary atmospheres (1244); Chemical abundances (224);

Abundance ratios (11)

Supporting material: figure set

\section{Introduction}

The Kepler Space Mission (Borucki et al. 2010) has shown that super-Earths/mini-Neptunes are among the most abundant type of planet (Fressin et al. 2013; Fulton et al. 2017). However, their formation history, internal and atmospheric composition, and chemistry remain poorly understood due to their relatively small size and the presence of clouds (Benneke et al. 2019a; Madhusudhan et al. 2020). After the Kepler mission, the Transiting Exoplanet Survey Satellite (Ricker et al. 2015), has provided more super-Earths/mini-Neptunes for characterization and future study of atmospheric composition (Chouqar et al. 2020; Fortenbach \& Dressing 2020).

As a successor to the Hubble Space Telescope (HST), the James Webb Space Telescope (JWST) -with its larger collection area-will allow for higher resolution and increased wavelength coverage to probe the atmospheric compositions of transiting super-Earths/mini-Neptunes.

With a growing list of potentially habitable planets, the search for biosignatures is the next logical step in exoplanet studies. Biosignatures such as $\mathrm{O}_{2}$ and $\mathrm{CH}_{4}$ are familiar to Earth-like planets (Des Marais et al. 2002). However, there are a limited number of Earth-sized planets (e.g., TRAPPIST-1 d

\footnotetext{
LSSTC DSFP Fellow.
}

and e, Gillon et al. 2017) that can be accessed by JWST. Moreover, the observation is very challenging in terms of the signals $(\sim 10 \mathrm{ppm})$ and thus the required telescope time (Lustig-Yaeger et al. 2019; Suissa et al. 2020).

Instead, we focus on gas dwarf planets (Buchhave et al. 2014), which we define as super-Earths/mini-Neptunes that have hydrogen-dominated atmospheres with radii larger than $1.7 R_{\oplus}$ and extend to $3.9 R_{\oplus}$. Beyond $1.7 R_{\oplus}$, planets lie in the second bimodal distribution of the radius valley and are more likely to have a gaseous envelope (Fulton et al. 2017; Van Eylen et al. 2018). Gas dwarf planets are more amenable targets than Earth-like planets for transit observations because of larger radii. In addition, gas dwarf planets have larger atmosphere scale height because of the lower mean molecular weight (MMW) due to the H-dominate atmosphere, which further increases the transit signals.

Because of the H-dominated atmosphere, gas dwarf planets have different atmospheric chemistry. The dominance of hydrogen creates a reducing chemistry, causing other elements to preferentially react with hydrogen to produce molecules such as water $\left(\mathrm{H}_{2} \mathrm{O}\right)$, ammonia $\left(\mathrm{NH}_{3}\right)$, and methane $\left(\mathrm{CH}_{4}\right)$. This is in contrast with the oxidizing chemistry that exists on the

\footnotetext{
8 Beyond $3.9 R_{\oplus}$, these objects are considered ice or gas giants based on the host star metallicities (Buchhave et al. 2014).
} 
inhabited Earth. We therefore expect different biosignatures in gas dwarf planets.

Seager et al. (2013b) first proposed $\mathrm{NH}_{3}$ as a biosignature gas in a $\mathrm{H}_{2}$ and $\mathrm{N}_{2}$ dominated atmosphere, nicknamed a cold Haber world, the reaction is as follows:

$$
3 \mathrm{H}_{2}+\mathrm{N}_{2} \longrightarrow 2 \mathrm{NH}_{3} \text {. }
$$

$\mathrm{NH}_{3}$ is a strong candidate as a biosignature for the following reasons (Seager et al. 2013b): first, the reaction that produces $\mathrm{NH}_{3}$ from $\mathrm{N}_{2}$ and $\mathrm{H}_{2}$ is exothermic, i.e., releasing energy that can be harnessed by life to support metabolism, second, this reaction requires high temperatures $(>450 \mathrm{~K})$ and high pressures ( $>10$ bar) in abiotic environments, and therefore the existence of $\mathrm{NH}_{3}$ at low temperatures and pressures in the upper atmosphere implies the existence of a reaction catalyst, potentially developed by life for metabolic processes, and third $\mathrm{NH}_{3}$ is easily destructible in photochemistry and volcanic environments. Therefore, any $\mathrm{NH}_{3}$ has to be replenished by certain productive processes that potentially involve life.

While $\mathrm{NH}_{3}$ is a promising biosignature for gas dwarf planets, the detection of $\mathrm{NH}_{3}$ only provides a necessary condition for an inhabited cold Haber world and sets the stage for follow-up observational work to confirm the detection and theoretical work to exhaust the chemical reaction network in order to ensure the production of $\mathrm{NH}_{3}$ is only made possible by life. The extreme challenge in carrying out these efforts has been highlighted in the recent controversy on the detection of $\mathrm{PH}_{3}$ in the atmosphere of Venus (Greaves et al. 2020, 2021; Snellen et al. 2020; Villanueva et al. 2020; Akins et al. 2021; Lincowski et al. 2021; Thompson 2021).

Along the same cautionary note, gas dwarfs are generally not considered habitable unless there is a substantial ocean layer with a relative thin atmosphere (Madhusudhan et al. 2020; Scheucher et al. 2020; Mousis et al. 2020; Hu 2021; Nixon \& Madhusudhan 2021). Such ocean/Hycean worlds can exist, e.g., K2-18 b and TOI-270 d (Madhusudhan et al. 2020; Madhusudhan et al. 2021). However, we discuss gas dwarfs in this paper because there may be possibilities for floating microbial life (Seager et al. 2021). Biosignature aside, the investigation of physical and chemical conditions of hydrogendominated atmospheres is in itself an exciting research subject.

In this paper, we study the feasibility of using JWST to search for $\mathrm{NH}_{3}$ in the atmospheres of gas dwarf planets. In Section 2, we describe the selection criteria for the targets in this study. We describe the model and simulations of transmission and emission spectra of targets in Section 3. We discuss the JWST simulations and introduce a detection metric for $\mathrm{NH}_{3}$ in Section 4, and discuss our results and various factors that affect the detection of ammonia in Section 5. To validate the detection metric that we use in this work, we provide examples of atmospheric retrieval in Section 6 to show that $\mathrm{NH}_{3}$ and $\mathrm{H}_{2} \mathrm{O}$ can be detected in optimal conditions. Discussions and conclusions are provided in Sections 7 and 8.

\section{Sample Selection}

We have compiled a list of targets that would be optimal for observations following the launch of JWST. We use the NASA Exoplanet Archive (NEA) ${ }^{9}$ and the following selection criteria: (1) planet radii between 1.7 and $3.4 R_{\oplus}$; (2) equilibrium temperature $\left(T_{\text {eq }}\right)$ below $450 \mathrm{~K}$; and (3) distance within $50 \mathrm{pc}$.

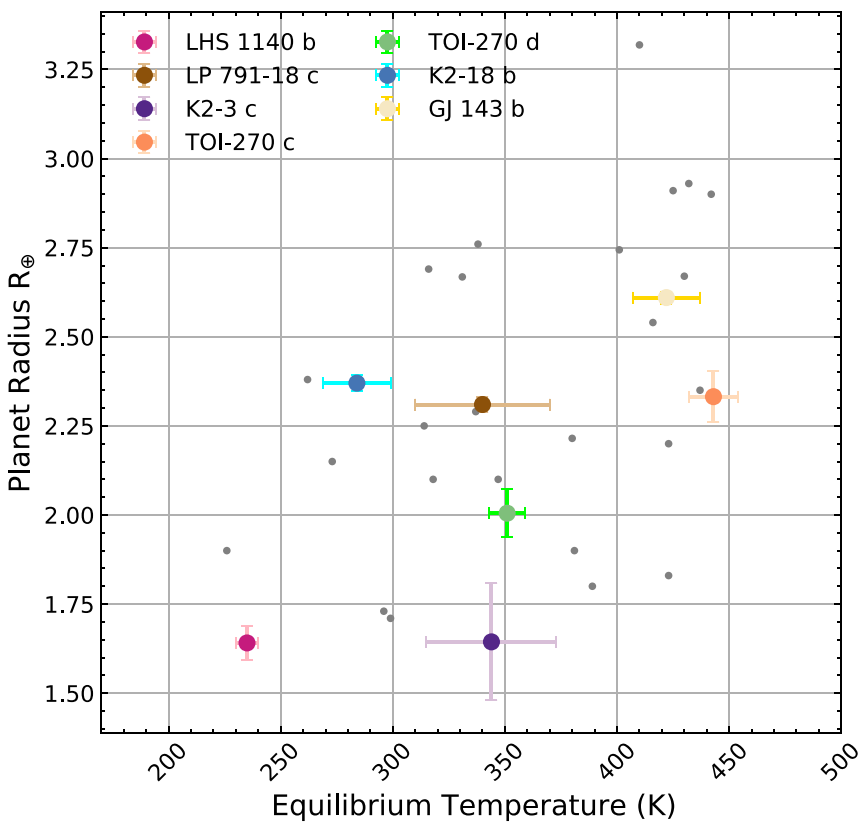

Figure 1. Planet radius $\left(R_{\oplus}\right)$ vs. equilibrium temperature (K) for LHS $1140 \mathrm{~b}$, LP 791-18 c, K2-3 c, K2-18 b, TOI-270 c, TOI-270 d, and GJ 143 b. Lighter gray circles are those with distances $>50 \mathrm{pc}$ but still have radii between 1.7 and 3.4 Earth radii and $T_{\text {eq }}<450 \mathrm{~K}$. Our seven colored targets are those that meet our full selection criteria in Section 2.

The $1.7 R_{\oplus}$ radius cut makes it more likely that our candidates have a gaseous envelope (Rogers 2015). Additionally, all but two planets in our sample live above the perioddependent radius gap (Van Eylen et al. 2018) further suggesting that these planets are indeed gas dwarfs/subNeptunes. The upper limit on radius reduces the likelihood that planets have a sufficiently high surface pressure to produce abiotic $\mathrm{NH}_{3}$. We note, however, the surface pressure can vary by orders of magnitude depending on planet internal structure and atmospheric composition (Madhusudhan et al. 2020). In Jupiter, the threshold pressure for chemical production of $\mathrm{NH}_{3}$ is $\sim 1000$ bar (Prinn \& Olaguer 1981). The upper limit of $\mathrm{T}_{\mathrm{eq}}$ at $450 \mathrm{~K}$ is where liquid water can exist at 10 bar pressure (Chaplin 2019). Lastly, we select only nearby systems within $50 \mathrm{pc}$ to ensure adequate flux from the star and planet.

Based on our selection criteria, we select seven targets for this work: LHS $1140 \mathrm{~b}, \mathrm{~K} 2-3 \mathrm{c}$, TOI-270 c, TOI-270 d, K2-18 b, GJ 143 b, and LP 791-18 c (Figure 1). We summarize planetary and stellar parameters used in this study in Table 1.

\section{Simulating Transmission and Emission Spectra}

We use the Python package, petitRADTRANS ${ }^{10}$ (Mollière et al. 2019), which calculates emission and transmission spectra of exoplanets. The emission and transmission spectra are produced through the implementation of a radiative transfer code. The atmosphere is assumed to be plane-parallel and in local thermodynamic equilibrium. The open-source radiative transfer code allows for modification of pressure-temperature (P-T) profile (Section 3.1), atmospheric composition and MMW (Section 3.2), surface gravity and planet radius 
Table 1

Planetary and Stellar Parameters of Targets Selected for This Work

\begin{tabular}{|c|c|c|c|c|c|c|c|}
\hline & LHS $1140 \mathrm{~b}$ & K2-3 c & TOI-270 $c^{d}$ & TOI-270 d & $\mathrm{K} 2-18 \mathrm{~b}$ & GJ $143 b^{\mathrm{e}}$ & LP 791-18 $\mathrm{c}^{\mathrm{g}}$ \\
\hline$\overline{M_{p}\left(M_{\oplus}\right)}$ & $6.96 \pm 0.89^{b}$ & $2.14_{-1.04}^{+1.08}(7)$ & $6.14 \pm 0.38$ & $4.78 \pm 0.46$ & $8.92_{-1.60}^{+1.70}(11)$ & $22.7_{-1.9}^{+2.2}$ & $5.96(6)^{\mathrm{f}}$ \\
\hline$R_{p}\left(R_{\oplus}\right)$ & $\begin{array}{c}1.635 \pm \\
0.046^{\mathrm{h}}(2)\end{array}$ & $1.618_{-0.207}^{+0.212 \mathrm{i}}(7)$ & $2.332 \pm 0.072$ & $2.005 \pm 0.007$ & $2.3 \pm 0.22(11)$ & $2.61_{-0.16}^{+0.17}$ & $2.31 \pm 0.25$ \\
\hline$T_{\text {eq }}(\mathrm{K})$ & $235 \pm 5(1)$ & $344 \pm 29(8)$ & $443 \pm 11$ & $351 \pm 8$ & $284 \pm 15(11)$ & $422_{-14}^{+15}$ & $370 \pm 30$ \\
\hline Distance (pc) & $14.98 \pm 0.01$ & $44.07 \pm 0.10$ & \multicolumn{2}{|c|}{$22.458 \pm 0.0059$} & $38.02 \pm 0.07$ & $16.32 \pm 0.0071$ & $26.4927_{-0.0638}^{+0.0640}$ \\
\hline$K \operatorname{band}_{s}(\mathrm{mag})^{\mathrm{c}}$ & $8.821 \pm 0.024$ & $8.561 \pm 0.023$ & \multicolumn{2}{|c|}{$8.251 \pm 0.029$} & $8.99 \pm 0.02$ & $5.524 \pm 0.031$ & $10.644 \pm 0.023$ \\
\hline$t_{14}(\mathrm{hr})$ & $\begin{array}{c}2.055 \pm \\
0.0046(2)\end{array}$ & $3.38 \pm 0.12(4)$ & $1.658_{-0.012}^{+0.015}(10)$ & $\begin{array}{c}2.14 \pm \\
0.018(10)\end{array}$ & $2.663_{-0.027}^{+0.023}(14)$ & $3.20_{-0.038}^{+0.041}$ & $1.208_{-0.046}^{+0.056}$ \\
\hline $\mathrm{Fe} / \mathrm{H}(\mathrm{dex})$ & $-0.24 \pm 0.10$ & $-0.32 \pm 0.13$ & \multicolumn{2}{|c|}{$-0.20 \pm 0.12$} & $0.12 \pm 0.16(11)$ & $0.003 \pm 0.06$ & $-0.09 \pm 0.19$ \\
\hline
\end{tabular}

Notes.

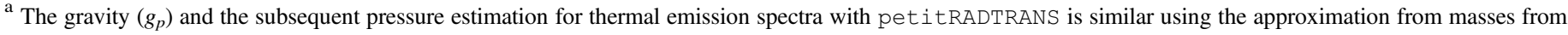
NASA Exoplanet Archive.

${ }^{\text {b }}$ Lillo-Box et al. (2020) find a RV measurement mass of $6.48 \pm 0.46 M_{\oplus}$.

c All values from Two Micron All Sky Survey (2MASS), unless otherwise noted (Cutri et al. 2003).

d All values from Van Eylen et al. (2021) unless otherwise noted.

e All values from Dragomir et al. (2019) unless otherwise noted.

${ }^{\text {f }}$ Crossfield et al. (2019) estimate a mass of $7 M_{\oplus}$.

g All values from Crossfield et al. (2019) unless otherwise noted.

h The radius of LHS $1140 \mathrm{~b}$ is very close to the $1.7 R_{E}$ and it is one of the most well-characterized potential gas dwarf planets, so we include it in this study.

${ }^{\mathrm{i}}$ We include K2-3 c because its radius uncertainty overlaps with the Van Eylen et al. (2018) radius gap and our $1.7 R_{\oplus}$ cutoff.

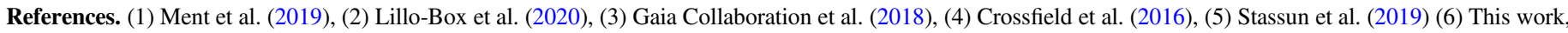

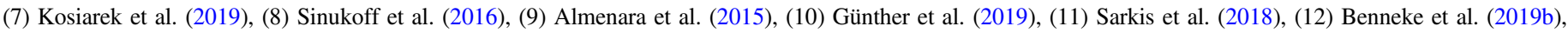
(13) Crossfield et al. (2016), (14) Benneke et al. (2017).

(Section 3.3). The code can account for the effects of clouds, scattering, and collision induced absorption.

There are two resolution modes available: low $(R=$ $\left.\frac{\lambda}{\Delta \lambda}=1000\right)$ and high $\left(R=\frac{\lambda}{\Delta \lambda}=10^{6}\right)$ modes. We utilize the low resolution mode, given that the Mid-Infrared Instrument (MIRI) LRS and Near-Infrared Spectrograph (NIRSpec), and NIRISS (SOSS) modes have resolutions of $\sim 100,100-2700$, and 700 , respectively. In this study, we test a variety of cases for our sample: (1) no clouds, high-MMW emission spectra, (2) no clouds, low-MMW emission spectra, (3) no clouds, high-MMW transmission spectra, (4) no clouds, low-MMW transmission spectra, and (5) cloud deck at $0.01 \mathrm{bar}, 0.1 \mathrm{bar}$, and $1 \mathrm{bar}$, lowMMW transmission spectra.

\subsection{P-T Profiles}

For emission spectroscopy the P-T profiles for our targets are adjusted and based on the $\mathrm{P}-\mathrm{T}$ profile of Earth. According to Seager et al. (2013a) - who approximates a P-T profile for a cold Haber world with a $T_{\mathrm{eq}}=290 \mathrm{~K}$-the precise P-T structure of the atmosphere is less important than photochemistry for a first order description of biosignatures in $\mathrm{H}_{2}$ rich atmospheres. We utilize public atmospheric data available from Public Domain Aeronautical Software (PDAS) to produce a P-T profile for Earth, ${ }^{11}$ and shift the profile based on the planet surface pressure so that 1 bar atmospheres matches the equilibrium temperature of the targets.

We use an isothermal P-T profile for the transmission spectroscopy. Unlike emission spectroscopy, an isothermal $\mathrm{P}-\mathrm{T}$ profile will not produce a featureless transmission spectrum.

\footnotetext{
${ }^{11}$ http://www.pdas.com/atmosdownload.html
}

However, isothermal profile may be overly simplified and can introduce bias in retrieval analysis (Rocchetto et al. 2016).

\subsection{Atmospheric Composition}

We calculate the volume mixing ratio (VMR) using values for a cold Haber world (Seager et al. 2013b) and the VMRs are reported in Tables 2 and 3. We assume the same chemistry of a cold Haber world to focus on the effects of temperature on the magnitude of transmission spectrum. The VMR is calculated using the following equation:

$$
\mathrm{VMR}=\frac{n_{i}}{\Sigma n_{i}}
$$

where $n_{i}$ is the mixing ratio from (Seager et al. 2013b) for a given species at 1 bar pressure. The species with opacity information in petitRADTRANS are: $\mathrm{H}_{2} \mathrm{O}, \mathrm{CO}_{2}, \mathrm{CH}_{4}, \mathrm{H}_{2}$, $\mathrm{CO}, \mathrm{HCN}, \mathrm{OH}$, and $\mathrm{NH}_{3}$. In addition, we include $\mathrm{H}_{2}$ and $\mathrm{He}$ for collision-induced absorption and $\mathrm{N}_{2}$ as a filler gas.

The VMRs from Seager et al. (2013b) are summed and normalized by dividing by the summation so the total VMR adds to 1.0 .

Since petitRADTRANS takes in mass mixing ratio (MMR), we calculate MMR as follows:

$$
\mathrm{MMR}_{i}=\frac{\mu_{i}}{\mu} \mathrm{VMR}_{i}
$$

where $\mu_{i}$ is the mass of the species in atomic unit, $\mu$ is the MMW for the atmosphere in atomic unit, and $\mathrm{VMR}_{i}$ is the volume mixing 
Table 2

Species used for petitRADTRANS to Generate Synthetic Spectra with 25\% $\mathrm{H}_{2}$ and $75 \% \mathrm{~N}_{2}$

\begin{tabular}{lcc}
\hline \hline Species & VMR & MMR \\
\hline $\mathrm{H}_{2} \mathrm{O}$ & $9.23 \mathrm{e}-07$ & $8.24 \mathrm{e}-07$ \\
$\mathrm{CO}_{2}$ & $2.92 \mathrm{e}-09$ & $6.37 \mathrm{e}-09$ \\
$\mathrm{CH}_{4}$ & $2.92 \mathrm{e}-08$ & $2.31 \mathrm{e}-08$ \\
$\mathrm{H}_{2}$ & $2.30 \mathrm{e}-01$ & $2.29 \mathrm{e}-02$ \\
$\mathrm{CO}$ & $9.23 \mathrm{e}-10$ & $1.28 \mathrm{e}-09$ \\
$\mathrm{OH}$ & $9.23 \mathrm{e}-16$ & $7.78 \mathrm{e}-16$ \\
$\mathrm{HCN}$ & $9.23 \mathrm{e}-10$ & $1.23 \mathrm{e}-09$ \\
$\mathrm{NH}_{3}$ & $3.69 \mathrm{e}-06$ & $3.11 \mathrm{e}-06$ \\
$\mathrm{He}$ & $7.69 \mathrm{e}-02$ & $1.52 \mathrm{e}-02$ \\
$\mathrm{~N}_{2 \mathrm{a}}$ & $6.92 \mathrm{e}-01$ & $9.61 \mathrm{e}-01$ \\
\hline
\end{tabular}

Note.

${ }^{\mathrm{a}} \mathrm{N}_{2}$ has no rotational-vibrational transitions, so there are no spectral signatures visible at infrared wavelengths, so this feature is not available in petitRADTRANS but is used to determine mean molecular weight of atmosphere.

Table 3

Species Used for petitRADTRANS to Generate Synthetic Spectra with $90 \%$ $\mathrm{H}_{2}$ and $10 \% \mathrm{~N}_{2 \mathrm{a}}$ Atmosphere

\begin{tabular}{lcc}
\hline \hline Species & VMR & MMR \\
\hline $\mathrm{H}_{2} \mathrm{O}$ & $9.17 \mathrm{e}-07$ & $3.62 \mathrm{e}-06$ \\
$\mathrm{CO}_{2}$ & $2.90 \mathrm{e}-09$ & $2.81 \mathrm{e}-08$ \\
$\mathrm{CH}_{4}$ & $2.90 \mathrm{e}-08$ & $1.02 \mathrm{e}-07$ \\
$\mathrm{H}_{2}$ & $8.25 \mathrm{e}-01$ & $3.62 \mathrm{e}-01$ \\
$\mathrm{CO}$ & $9.17 \mathrm{e}-10$ & $5.64 \mathrm{e}-09$ \\
$\mathrm{OH}$ & $9.17 \mathrm{e}-16$ & $3.42 \mathrm{e}-15$ \\
$\mathrm{HCN}$ & $9.17 \mathrm{e}-10$ & $5.44 \mathrm{e}-09$ \\
$\mathrm{NH}_{3}$ & $3.66 \mathrm{e}-06$ & $1.37 \mathrm{e}-05$ \\
$\mathrm{~N}_{2 \mathrm{~b}}$ & $9.17 \mathrm{e}-02$ & $5.64 \mathrm{e}-01$ \\
$\mathrm{He}$ & $8.25 \mathrm{e}-02$ & $7.25 \mathrm{e}-02$ \\
\hline
\end{tabular}

Notes.

${ }^{a}$ We simply adopt the mixing ratios for gases other than $\mathrm{N}_{2}$ or $\mathrm{H}_{2}$, assuming no major change in the chemistry.

${ }^{b}$ has no rotational-vibrational transitions, so there are no spectral signatures visible at infrared wavelengths, so this feature is not available in petitRADTRANS but is used to determine mean molecular weight of atmosphere.

ratio. The MMW is calculated as

$$
\mu=\sum \mu_{i} \cdot \mathrm{VMR}_{i}
$$

\subsection{Radius and Surface Gravity}

Radius and surface gravity are also the input for petitRADTRANS. The radii are taken from the NASA Exoplanet Archive (Table 1). To calculate surface gravity, we also get masses from NEA, except for the mass of LP 791-18 c for which we use a mass-radius scaling relation (Crossfield et al.2019).

\subsection{Emission Spectroscopy}

With the inputs that are described in previous sections, we model the emission spectra $\left(f_{p}\right)$ as shown in Figure 2. The output flux unit for petitRADTRANS in mJy is converted to $f_{p} / f_{\star}$, which is the input for PandExo (Batalha et al. 2017) to generate simulated JWST data.

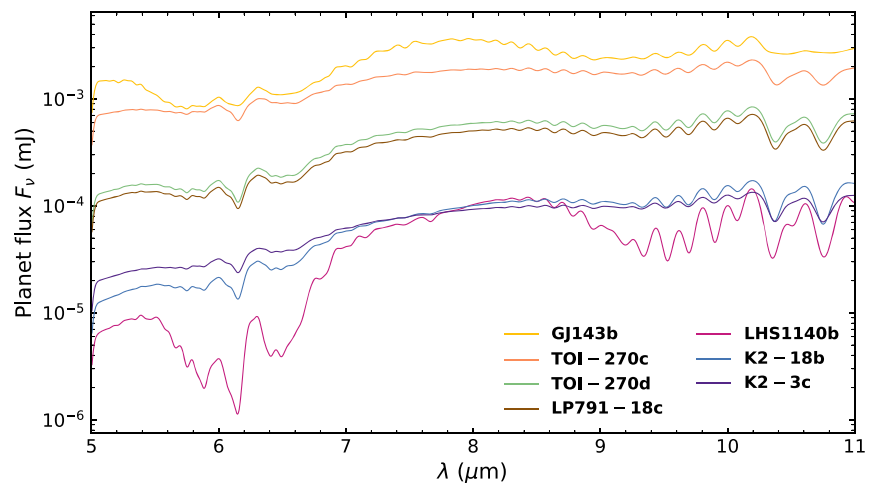

Figure 2. Synthetic emission spectra of select targets generated from petitRADTRANS: GJ 143 b, TOI-270 c, TOI-270 d, LHS 1140 b, K2-18 b, LP 791-18 c, and K2-3 c. The spectra have been convolved with a 1D Gaussian kernel down to $R \sim 100$ from $R \sim 1000$ to match the resolution of MIRI LRS.

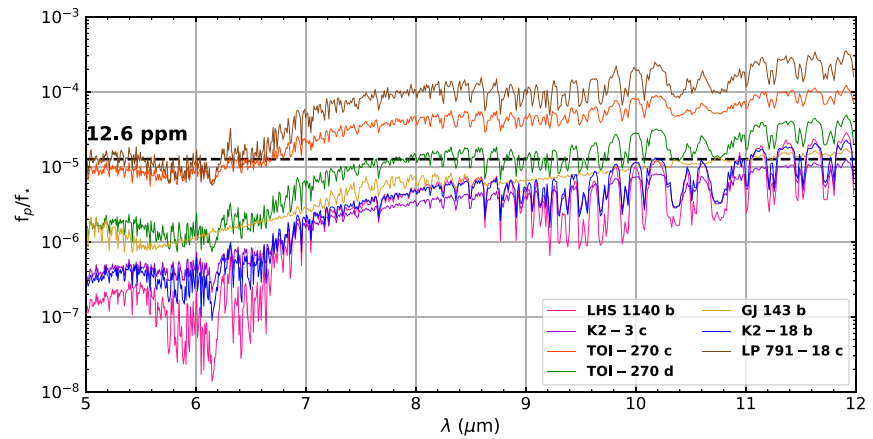

Figure 3. Simulated flux ratio $\left(f_{p} / f_{\star}\right)$ comparisons for LHS $1140 \mathrm{~b}, \mathrm{~K} 2-3 \mathrm{c}$, TOI-270 c, TOI-270 d, GJ 143 b, K2-18 b, and LP 791-18 c for a $90 \% \mathrm{H}_{2} / 10 \%$ $\mathrm{N}_{2}$, no clouds atmosphere. The dashed line represents the systemic noise limit for MIRI LRS for 10 transits, where the noise level shown (12.6 ppm) follows as $40 / \sqrt{N_{\text {obs }}}$, where $N_{\text {obs }}=10$.

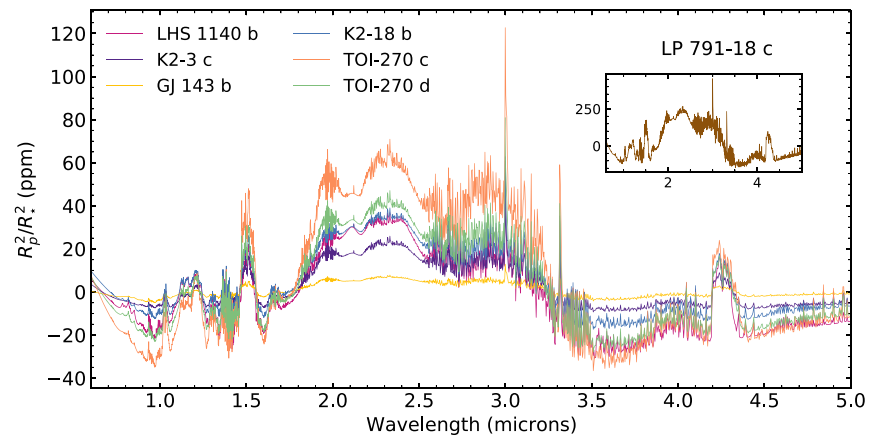

Figure 4. Transmission signal (minus offset) for a $90 \% \mathrm{H}_{2} / 10 \% \mathrm{~N}_{2}$, no clouds atmosphere for targets in our sample. LP 791-18 c has the highest transmission signal and is shown in a separate plot to the side.

To calculate stellar flux $\left(f_{\star}\right)$, we use the PHOENIX model grids (Husser et al. 2013). We resample the wavelength grid of the synthetic planet spectrum from petitRADTRANS onto the wavelength grid of the PHOENIX model to determine $f_{p} / f_{\star}$. Examples of planet-star contrast spectra are shown in Figure 3.

\subsection{Transmission Spectroscopy}

Some examples of the modeled transmission spectra are shown in Figure 4. The reference pressure for all targets is set 
Table 4

Summary of Instruments and Modes

\begin{tabular}{lcccc}
\hline \hline Instrument & Mode & Coverage & Resolution & Throughput $^{\mathrm{b}}$ \\
\hline NIRSpec & G235M & $1.7-3.0 \mu \mathrm{m}$ & 1000 & 0.5 \\
NIRSpec & G395M & $2.9-5.0 \mu \mathrm{m}$ & 1000 & 0.6 \\
NIRSpec & PRISM/CLEAR & $0.6-5.3 \mu \mathrm{m}$ & 100 & 0.5 \\
NIRISS & SOSS & $0.6-2.8 \mu \mathrm{m}$ & 700 & 0.3 \\
MIRI & LRS/slitless & $5-12 \mu \mathrm{m}$ & 100 & 0.3 \\
\hline
\end{tabular}

Notes.

${ }^{\text {a }}$ NIRSpec/G235H has a higher resolution $(R \sim 2700)$ and comparable sensitivity but has a gap in the wavelength coverage so it is omitted from this study.

${ }^{\mathrm{b}}$ Estimated peak throughput values from JWST ETC version 1.6

to $P_{0}=1.0$ bar and the planet radius and surface gravity values (Table 1) are given at $P_{0}$ in petitRADTRANS.

\section{Simulating JWST Observations}

\subsection{Considered Instruments}

We consider the NIRSpec, MIRI, and NIRISS instruments for this work. ${ }^{12}$ A summary of the instrument specifications is provided in Table 4. These instruments allow for a range of wavelengths that cover major $\mathrm{NH}_{3}$ features near 1.0-1.5, 2.0, $2.3,3.0,5.5-6.5$, and $10.3-10.8 \mu \mathrm{m}$, and spectral features from other molecular species such as $\mathrm{CO}, \mathrm{CO}_{2}, \mathrm{HCN}$, and $\mathrm{CH}_{4}$. NIRCam has lower throughput than NIRSpec, MIRI, and NIRISS so we omit this instrument from this study.

\subsection{Eclipse Spectroscopy with MIRI}

Ammonia has major absorption features at 10.3-10.8 $\mu \mathrm{m}$, so we test the capabilities of MIRI LRS (Kendrew et al. 2015) to detect this feature. We exclude the use of MIRI MRS because although this mode has a higher resolution, it has a lower throughput than MIRI LRS (Glasse et al. 2015). We note that MIRI MRS has been considered in other nontransiting exoplanet studies (Snellen et al. 2017). The use of MIRI MRS for transiting exoplanets is still possible; capabilities of this mode will be further investigated in Cycle 1 (Kendrew et al. 2018; Deming et al. 2021).

We use PandExo (Batalha et al. 2017) to simulate observations for our targets. We assume a stack of 10 eclipse observations, an $80 \%$ detector saturation level, and a floor noise of $40 \mathrm{ppm}$. The floor noise is set following Chouqar et al. (2020), adopting a value of $40 \mathrm{ppm}$ between the $30 \mathrm{ppm}$ reported in Beichman et al. (2014) and 50 ppm reported in Greene et al. (2016). The noise floor in PandExo is only for one transit, and goes as $1 / \sqrt{N}$ for stacking $N$ eclipses.

To further set up a run in PandExo, we input our simulated $f_{p} / f_{\star}$ spectra and PandExo uses the incorporated PHOENIX model library (Husser et al. 2013) as the stellar input for the simulated observations based on the effective temperature, surface gravity, $K$-band magnitude, and metallicity $[\mathrm{Fe} / \mathrm{H}]$ for each target host star.

\footnotetext{
12 All assumptions on the performance of these instruments are based on prelaunch, ground-test data, and models.
}

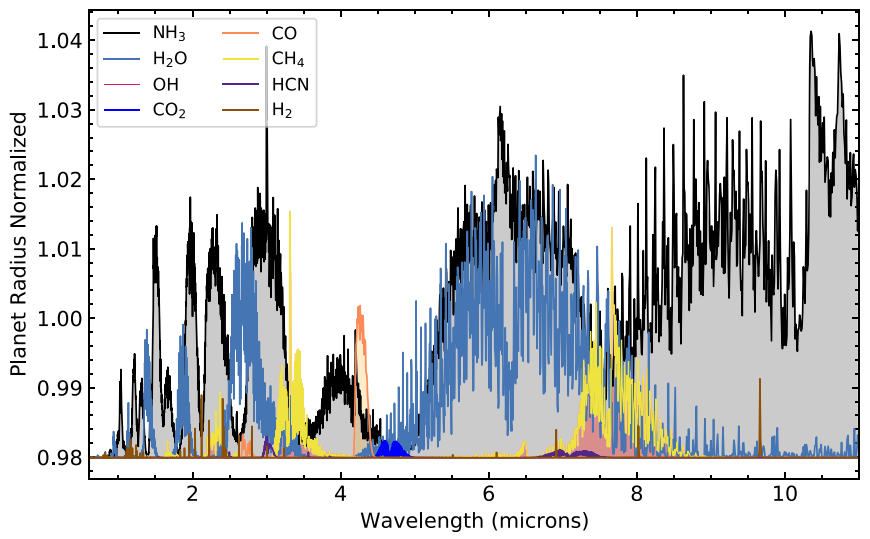

Figure 5. Near and mid-infrared wavelength ranges of major features of $\mathrm{NH}_{3}$ $\mathrm{H}_{2} \mathrm{O}, \mathrm{OH}, \mathrm{CO}, \mathrm{CO}_{2}, \mathrm{CH}_{4}, \mathrm{HCN}$, and $\mathrm{H}_{2}$.

\subsection{Detection Metric for Transmission Spectroscopy}

We define the significance of spectral feature detection using a signal to noise ratio $(\mathrm{S} / \mathrm{N})$ :

$$
\mathrm{S} / \mathrm{N}=\frac{\left(R_{p} / R_{\star}\right)^{2}-\overline{\left(R_{p} / R_{\star}\right)^{2}}}{\sigma_{\left(R_{p} / R_{\star}\right)^{2}}},
$$

where $\left(R_{p} / R_{\star}\right)^{2}$ is the transmission signal from petitRADTRANS, $\overline{\left(R_{p} / R_{\star}\right)^{2}}$ is median of the transmission signal from petitRADTRANS ${ }^{13}$ and $\sigma_{\left(R_{p} / R_{\star}\right)^{2}}$ is the uncertainty.

Following Wunderlich et al. (2021) and Chouqar et al. (2020) we note ammonia features in the near and mid-infrared have other spectral features that overlap and can obscure these feature (Figure 5). For example, the $2.0 \mu \mathrm{m} \mathrm{NH} \mathrm{NH}_{3}$ feature overlaps with $\mathrm{H}_{2} \mathrm{O}$ and $\mathrm{H}_{2}-\mathrm{H}_{2}$ features. In the mid-infrared the 10.3-10.8 $\mu \mathrm{m} \mathrm{NH}_{3}$ feature overlaps with $\mathrm{H}_{2}-\mathrm{H}_{2}$ (Wunderlich et al. 2021).

Similar to Chouqar et al. (2020), we neglect the complications from these overlapping features of $\mathrm{H}_{2} \mathrm{O}, \mathrm{NH}_{3}$ and other species. Our detection metric focuses on the $\mathrm{S} / \mathrm{N}$ for detecting any spectral features, whether or not they are overlapped. The metric will help in determining the number of transits to significantly detect potential biosignatures for given atmospheric compositions. In addition, we show in Section 6 that $\mathrm{H}_{2} \mathrm{O}$ and $\mathrm{NH}_{3}$ can be independently detected and constrained in atmospheric retrieval analyses despite overlapping spectral features.

\subsection{Transmission Spectroscopy with NIRSpec and NIRISS}

To simulate JWST transmission spectra, we utilize the Near InfrarRed Spectrograph (NIRSpec) instrument with the G235M and $\mathrm{G} 395 \mathrm{M}$ modes which covers the wavelength ranges of 1.7-3.0 $\mu \mathrm{m}$ and 2.9-5.0 $\mu \mathrm{m}$, respectively. NIRSpec/G395M mode has an expected floor noise of $25 \mathrm{ppm}$ (Kreidberg et al. 2014). We use a fixed number of 10 transits for our simulations with PandExo as with the MIRI LRS simulation.

We assume an optimistic noise floor level for NIRISS (SOSS) and the NIRSpec modes. Greene et al. (2016) notes that "The best HST WFC3 G141 observations of transiting systems to date have noise of the order of $30 \mathrm{ppm}$ (Kreidberg et al. 2014)...". The noise floor in PandExo is only for 1 transit, and goes as $1 / \sqrt{N}$ for stacking $N$ transits.

\footnotetext{
${ }^{13}$ We utilize wavelength ranges outside $1.0-3.5 \mu \mathrm{m}$ to calculate the median as the range below 1.0 and above 3.5 do not include any major $\mathrm{NH}_{3}$ absorption features.
} 

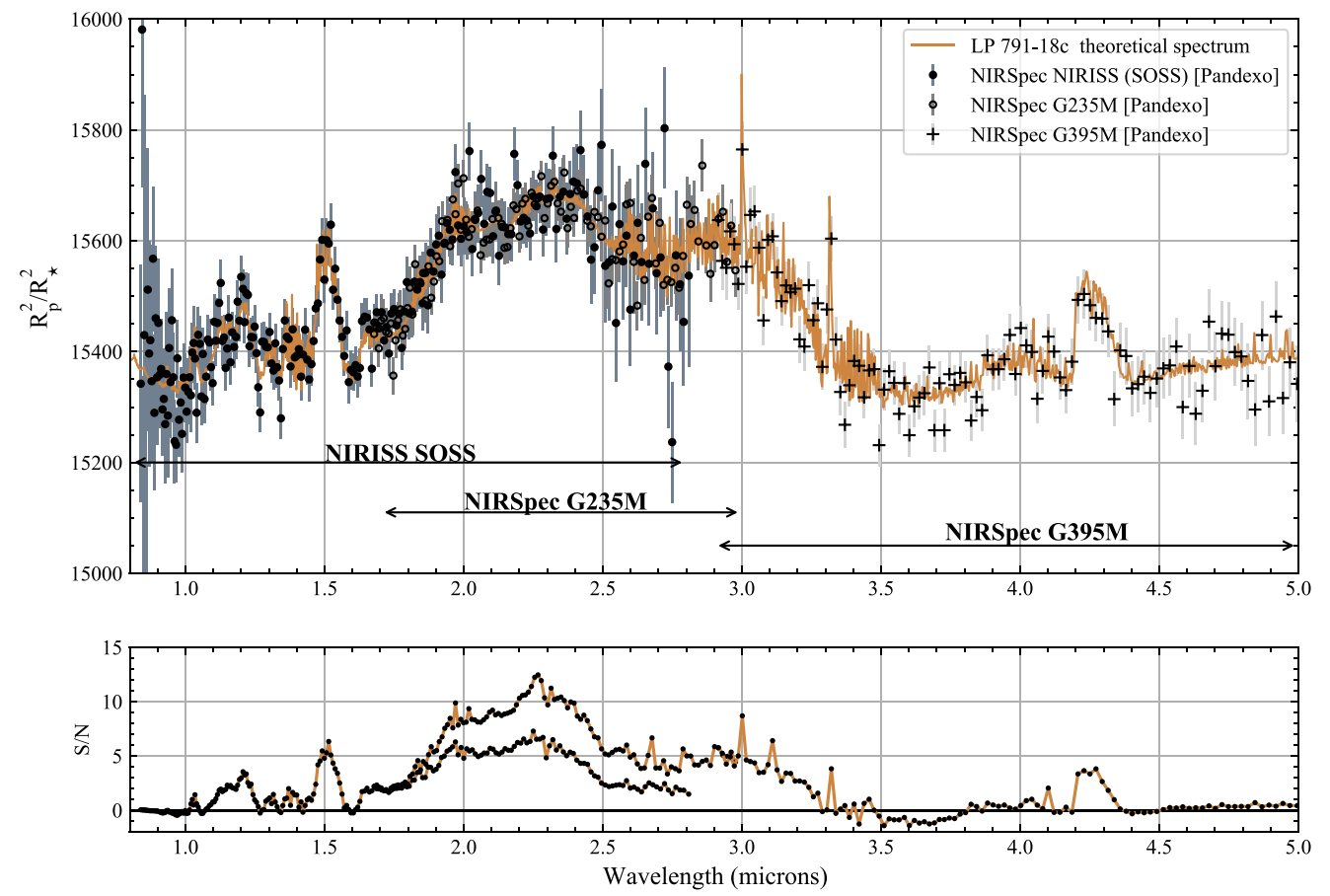

Figure 6. Top: simulated NIRSpec/G235M, NIRSpec/G395M, and NIRISS (SOSS) transmission spectrum of LP 791-18 c with 10 transits (MMW 4.46). Bottom: $\mathrm{S} / \mathrm{N}$ and deviation from flat line.

(The complete figure set (7 images) is available.)

We also consider the use of the Near Infrared Imager and Slitless Spectrograph (NIRISS) instrument in the Single Object Slitless Spectroscopy (SOSS) mode, which covers the $0.8-2.8 \mu \mathrm{m}$ range. This compliments the wavelength coverage for the NIRSpec/G235M and NIRSpec/G395M modes. An optimistic noise floor of $20 \mathrm{ppm}$ is adopted for NIRISS (SOSS) (Greene et al. 2016; Fortenbach \& Dressing 2020).

Pandexo simulations for these instruments for LHS $1140 \mathrm{~b}$, LP 791-18 c, TOI-270 d, LHS 1140 b, K2-18 b, K2-3 c, and GJ $143 \mathrm{~b}$ are provided in Figure 6.

\subsection{Transmission Spectroscopy with MIRI LRS}

We also simulate whether the 6.1 and 10.3-10.8 micron feature of ammonia is detectable with transmission spectroscopy with MIRI LRS, using our detection metric. We assume the same setup for PandExo as with Section 4.2.

\section{Main Results}

\subsection{Eclipse Spectroscopy with MIRI}

The majority of our targets are not feasible for eclipse spectroscopy with MIRI LRS (Figure 3). This is consistent with other studies that achieving the necessary flux contrast for significant detection of molecular features has proved to be very difficult with MIRI (Batalha et al. 2018; Chouqar et al. 2020). LP 791-18 c has the most promising flux contrast ratio but the emission signal is mostly overwhelmed by the photon noise, assuming 10 transits and $\sim 40 \mathrm{hr}$ of observing time (Figure 7). Similarly TOI-270 $\mathrm{c}$ and TOI-270 d are photon limited. LP 791-18 c, TOI-270 c, and TOI-270 d have an S/N of $0.3 \sigma, 0.2 \sigma$, and $0.1 \sigma$ for the $10.3-10.8 \mathrm{NH}_{3}$ feature. $\mathrm{K} 2-3 \mathrm{c}$, LHS $1140 \mathrm{~b}, \mathrm{~K} 2-18 \mathrm{~b}$, and GJ $143 \mathrm{~b}$ are limited by systemic noise as shown in Figure 3.
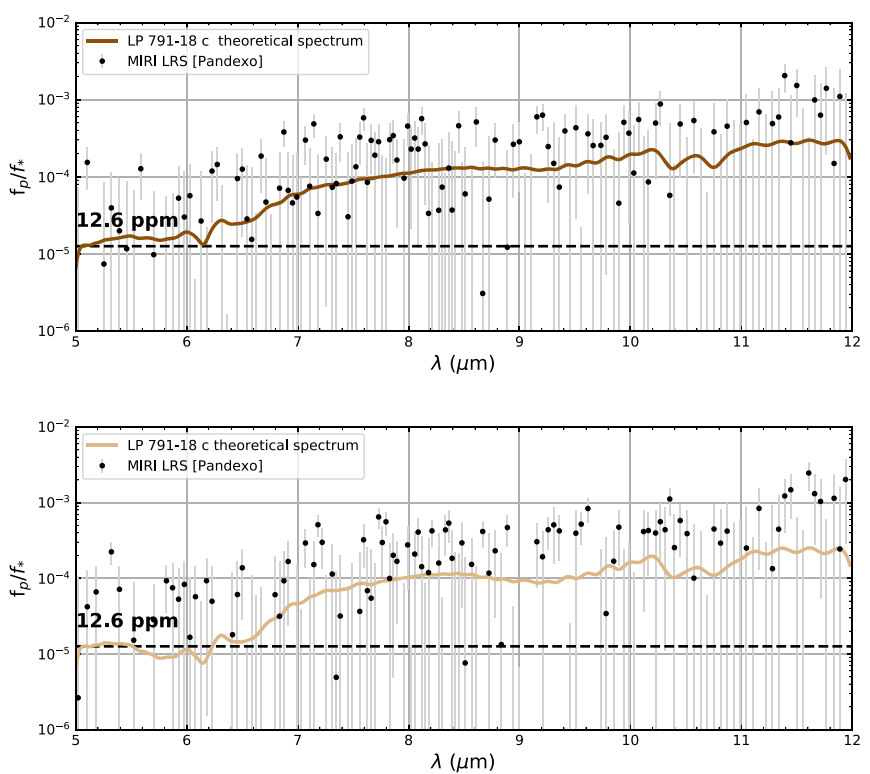

Figure 7. Simulated MIRI LRS emission spectra for 10 transits of LP 791-18 c The spectra from petitRADTRANS $(R \sim 1000)$ has been smoothed down to match the resolution of MIRI LRS $(R \sim 100)$. The dashed line represents the systemic noise limit for MIRI LRS for 10 transits, where the noise level shown (12.6 ppm) follows as $40 / \sqrt{N_{\text {obs }}}$, where $N_{\text {obs }}=10$. Top: high-MMW $\left(25 \% \mathrm{H}_{2}\right)$ atmosphere with no clouds. Bottom: low-MMW $\left(90 \% \mathrm{H}_{2}\right)$ atmosphere with no clouds.

\subsection{Transmission Spectroscopy with NIRISS, NIRSpec, and MIRI}

Based on our transmission spectroscopy detection metric (Equation (4)), we find that the NIRSpec/G235M, NIRSpec/ G395M, and NIRISS/SOSS modes are best suited to detect ammonia for our targets, with one exception-LP 791-18 c for 
Table 5

Saturation Limits for Our Targets

\begin{tabular}{|c|c|c|c|}
\hline Target & Instrument & Mode & Saturation \\
\hline \multirow[t]{5}{*}{ LHS $1140 \mathrm{~b}$} & NIRSpec & G235M & $\mathrm{N}$ \\
\hline & NIRSpec & G395M & $\mathrm{N}$ \\
\hline & NIRSpec & PRISM/CLEAR & $\mathrm{Y}$ \\
\hline & MIRI & LRS/slitless & $\mathrm{N}$ \\
\hline & NIRISS & SOSS & $\mathrm{N}$ \\
\hline \multirow{5}{*}{ LP $791-18 \mathrm{c}$} & NIRSpec & $\mathrm{G} 235 \mathrm{M}$ & $\mathrm{N}$ \\
\hline & NIRSpec & G395M & $\mathrm{N}$ \\
\hline & NIRSpec & PRISM/CLEAR & $\mathrm{N}$ \\
\hline & MIRI & LRS/slitless & $\mathrm{N}$ \\
\hline & NIRISS & SOSS & $\mathrm{N}$ \\
\hline \multirow[t]{5}{*}{$\mathrm{K} 2-3 \mathrm{c}$} & NIRSpec & G235M & $\mathrm{N}$ \\
\hline & NIRSpec & G395M & $\mathrm{N}$ \\
\hline & NIRSpec & PRISM/CLEAR & $\mathrm{Y}$ \\
\hline & MIRI & LRS/slitless & $\mathrm{N}$ \\
\hline & NIRISS & SOSS & $\mathrm{N}$ \\
\hline \multirow[t]{5}{*}{$\mathrm{K} 2-18 \mathrm{~b}$} & NIRSpec & G235M & $\mathrm{N}$ \\
\hline & NIRSpec & G395M & $\mathrm{N}$ \\
\hline & NIRSpec & PRISM/CLEAR & $\mathrm{Y}$ \\
\hline & MIRI & LRS/slitless & $\mathrm{N}$ \\
\hline & NIRISS & SOSS & $\mathrm{N}$ \\
\hline \multirow[t]{5}{*}{ TOI-270 c } & NIRSpec & $\mathrm{G} 235 \mathrm{M}$ & $\mathrm{N}$ \\
\hline & NIRSpec & G395M & $\mathrm{N}$ \\
\hline & NIRSpec & PRISM/CLEAR & $\mathrm{Y}$ \\
\hline & MIRI & LRS/slitless & $\mathrm{N}$ \\
\hline & NIRISS & SOSS & $\mathrm{N}$ \\
\hline \multirow[t]{5}{*}{ TOI-270 d } & NIRSpec & G235M & $\mathrm{N}$ \\
\hline & NIRSpec & G395M & $\mathrm{N}$ \\
\hline & NIRSpec & PRISM/CLEAR & $\mathrm{Y}$ \\
\hline & MIRI & LRS/slitless & $\mathrm{N}$ \\
\hline & NIRISS & SOSS & $\mathrm{N}$ \\
\hline \multirow[t]{5}{*}{ GJ 143 b } & NIRSpec & G235M & $\mathrm{Y}$ \\
\hline & NIRSpec & G395M & $\mathrm{Y}$ \\
\hline & NIRSpec & PRISM/CLEAR & $\mathrm{Y}$ \\
\hline & MIRI & LRS/slitless & $\mathrm{N}$ \\
\hline & NIRISS & SOSS & $\mathrm{Y}$ \\
\hline
\end{tabular}

which NIRSpec PRISM/CLEAR is optimal because this target does not saturate the detector (Table 5).

We compile a ranked list of targets based on transmission spectroscopy simulations for the NIRSpec/G235M, NIRSpec/ G395M, NIRISS/SOSS, and MIRI LRS modes with 10 transits. To compute the rank list (Table 6), we use six major absorption features of $\mathrm{NH}_{3}(1.5,2.0,2.3,3.0,6.1$, and $10.3-10.8 \mu \mathrm{m})$.

For the 1.5, 2.0, 2.3, 3.0, 6.1, and 10.3-10.8 $\mu \mathrm{m} \mathrm{NH}_{3}$ features we find the approximate central wavelength and use three data points [one centered on the approximate central wavelength and two adjacent data points] and compute the $\mathrm{S} / \mathrm{N}$ based on the average of the three data points.

For the final $\mathrm{S} / \mathrm{N}$ determination, we square each $\mathrm{S} / \mathrm{N}$ of the $\mathrm{NH}_{3}$ feature, take the summation, and take the square root of the summation (Equation (5)) to determine the ranking

$$
<\mathrm{S} / \mathrm{N}>=\sqrt{\sum_{i} \mathrm{~S} / \mathrm{N}_{i}^{2}}
$$

Table 6

$\mathrm{S} / \mathrm{N}$ of Major Near and Mid-infrared $\mathrm{NH}_{3}$ Transmission Features and Ranking of Targets

\begin{tabular}{|c|c|c|c|c|}
\hline Target & $\begin{array}{l}\text { Ammonia Feature } \\
(\mu \mathrm{m})\end{array}$ & $\begin{array}{l}\mathrm{S} / \mathrm{N} \\
(\sigma)\end{array}$ & $\begin{array}{c}<\mathrm{S} / \mathrm{N}> \\
(\sigma)\end{array}$ & Ranking \\
\hline TOI-270 c & $\begin{array}{c}1.5 \\
2.0 \\
2.3 \\
3.0 \\
6.1 \\
10.3-10.8\end{array}$ & $\begin{array}{l}5.0 \\
5.4 \\
5.1 \\
6.2 \\
5.5 \\
2.4\end{array}$ & 18.8 & 1 \\
\hline LP $791-18 \mathrm{c}$ & $\begin{array}{c}1.5 \\
2.0 \\
2.3 \\
3.0 \\
6.1 \\
10.3-10.8\end{array}$ & $\begin{array}{l}5.4 \\
5.4 \\
5.7 \\
6.0 \\
5.3 \\
1.6\end{array}$ & 18.4 & 2 \\
\hline TOI-270 d & $\begin{array}{c}1.5 \\
2.0 \\
2.3 \\
3.0 \\
6.1 \\
10.3-10.8\end{array}$ & $\begin{array}{l}3.9 \\
4.2 \\
4.0 \\
4.3 \\
3.7 \\
1.7\end{array}$ & 12.0 & 3 \\
\hline LHS $1140 \mathrm{~b}$ & $\begin{array}{c}1.5 \\
2.0 \\
2.3 \\
3.0 \\
6.1 \\
10.3-10.8\end{array}$ & $\begin{array}{l}2.5 \\
2.6 \\
2.7 \\
2.6 \\
2.3 \\
1.0\end{array}$ & 6.6 & 4 \\
\hline $\mathrm{K} 2-3 \mathrm{c}$ & $\begin{array}{c}1.5 \\
2.0 \\
2.3 \\
3.0 \\
6.1 \\
10.3-10.8\end{array}$ & $\begin{array}{l}2.1 \\
2.2 \\
2.0 \\
2.4 \\
2.3 \\
0.9\end{array}$ & 5.7 & 5 \\
\hline $\mathrm{K} 2-18 \mathrm{~b}$ & $\begin{array}{c}1.5 \\
2.0 \\
2.3 \\
3.0 \\
6.1 \\
10.3-10.8\end{array}$ & $\begin{array}{l}1.5 \\
1.6 \\
1.5 \\
1.7 \\
1.3 \\
0.5\end{array}$ & 3.7 & 6 \\
\hline GJ $143 b^{a}$ & $\begin{array}{c}1.5 \\
2.0 \\
2.3 \\
3.0 \\
6.1 \\
10.3-10.8\end{array}$ & $\begin{array}{l}\cdots \\
\cdots \\
\cdots \\
\cdots \\
1.5 \\
0.7\end{array}$ & 1.6 & 7 \\
\hline
\end{tabular}

Note.

${ }^{a}$ GJ 143 b does not saturate MIRI LRS; however, there is saturation with the NIRSpec, and NIRISS instruments (Table 5), and therefore it is not a target for JWST based on its brightness and is ranked last even though it could be observed at $3 \mu \mathrm{m}$ with the NIRCam grisms.

where $i$ indicates $\mathrm{NH}_{3} \lambda_{i}$ features at 1.5, 2.0, 2.3, 3.0, 6.1, and $10.3-10.8 \mu \mathrm{m}$.

TOI-270 $\mathrm{c}$ is best suited for atmospheric studies with JWST given the $\mathrm{S} / \mathrm{N}$ of detection features for $\mathrm{NH}_{3}$. On the other hand, GJ $143 \mathrm{~b}$ is the least favorable target given that it saturates the NIRSpec/G235M, NIRSpec/G395M, and NIRISS/SOSS modes due to the brightness of the host star (Table 5). 

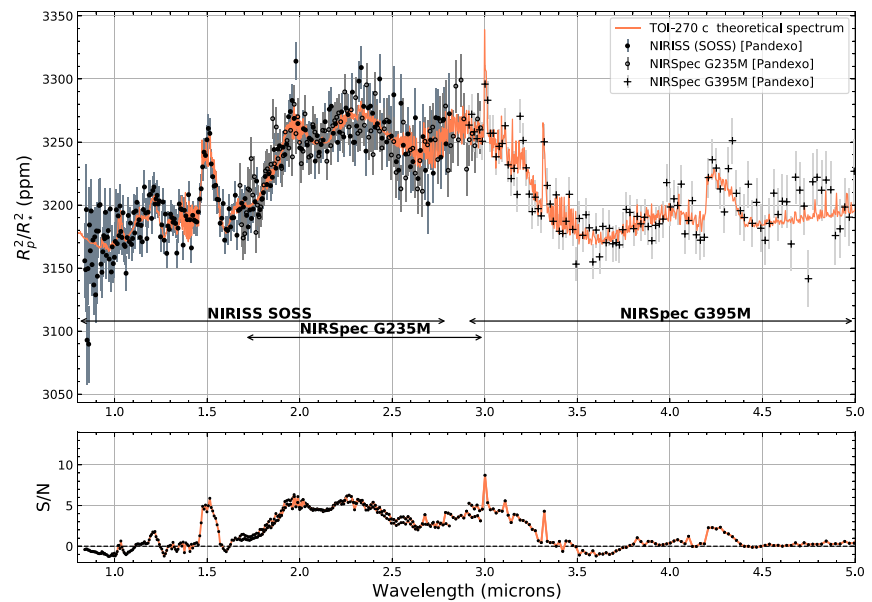

Figure 8. Simulated NIRSpec/G235M, NIRSpec/G395M, and NIRISS (SOSS) transmission spectrum of TOI-270 c with 10 transits and corresponding $\mathrm{S} / \mathrm{N}$ for low MMW (MMW 4.55) (Table 3).
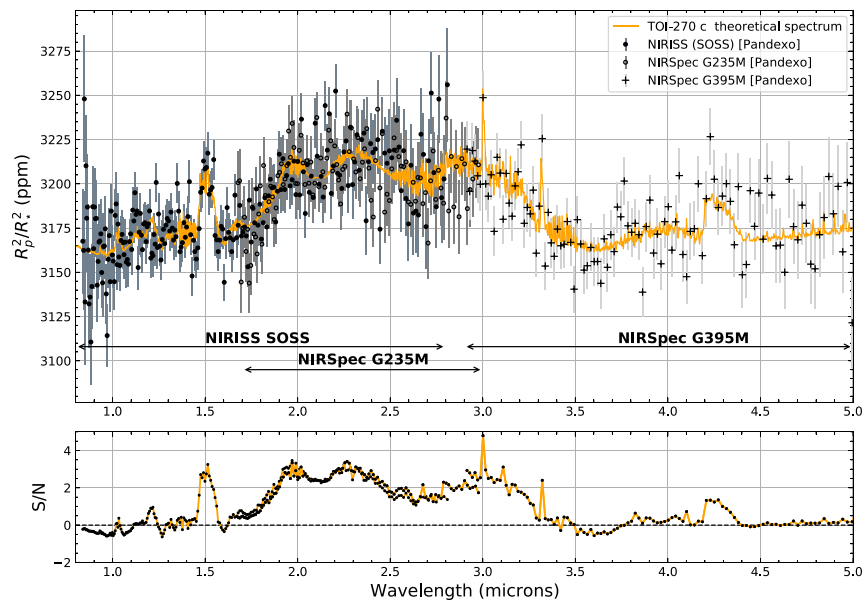

Figure 9. Simulated NIRSpec/G235M, NIRSpec/G395M, and NIRISS (SOSS) transmission spectrum of TOI-270 c with 10 transits and corresponding $\mathrm{S} / \mathrm{N}$ for the high-MMW case MMW $\sim 20.1$ (Table 2).
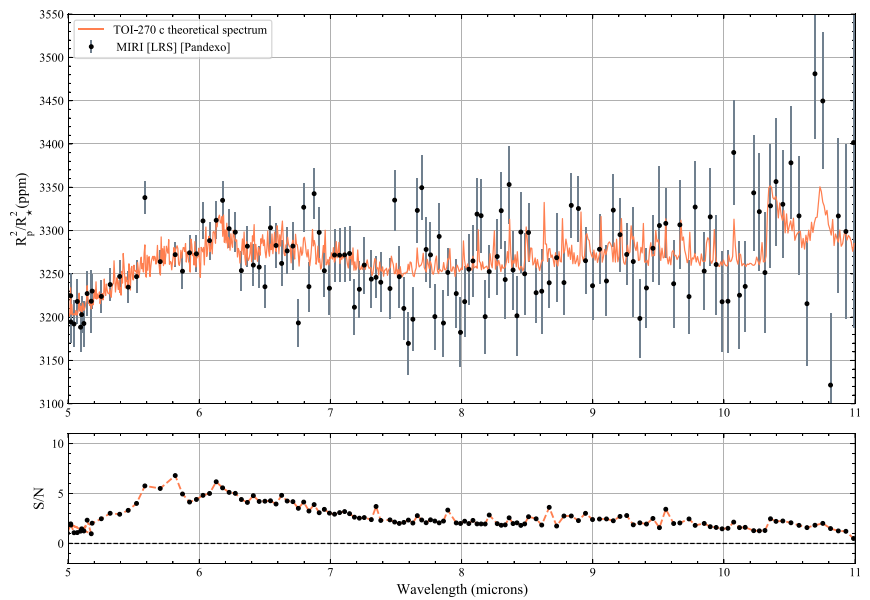

Figure 10. Simulated MIRI LRS transmission spectrum of TOI-270 $\mathrm{c}$ with 10 transits and corresponding $\mathrm{S} / \mathrm{N}$ for the low-MMW $\sim 4.6$ case.

We show a few examples of transmission spectra for TOI-270 c from Figures 8-10. As the $\mathrm{S} / \mathrm{N}$ scales inversely with $\mathrm{MMW}$, we find that the ideal observing conditions are atmospheres with a low-MMW and clear atmosphere (Figure 8) as opposed to a

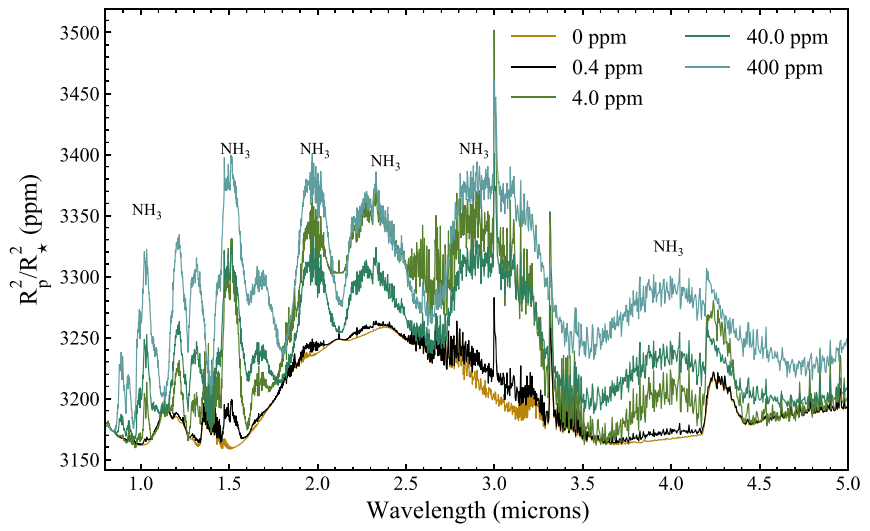

Figure 11. Theoretical transmission spectra of TOI-270 $\mathrm{c}$ with varying level of ammonia concentration: 0, 0.4, 4.0, 40, and $400 \mathrm{ppm}$.

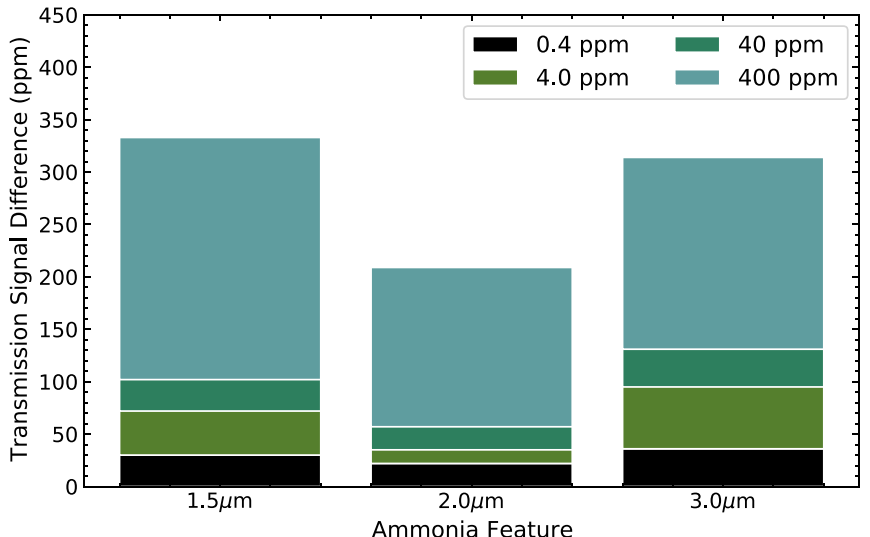

Figure 12. Transmission signal difference vs. major transmission features for $\mathrm{NH}_{3}(1.5,2.0,2.3$, and $3.0 \mu \mathrm{m})$ for varying concentrations of ammonia in the atmosphere of TOI-270 c $(0.4,4.0,40$, and $400 \mathrm{ppm})$. A higher concentration of ammonia in the atmosphere ( $400 \mathrm{ppm})$ produces the highest change in the transmission signal strength as compared to $0 \mathrm{ppm}$ of ammonia.

high-MMW atmosphere that produces a weaker transmission signal and therefore lower $\mathrm{S} / \mathrm{N}$ (Figure 9).

For MIRI transmission spectroscopy, We find that the 6.1 $\mu \mathrm{m} \mathrm{NH}$ is more promising for detection with transmission spectroscopy with MIRI LRS than the 10.3-10.8 $\mu \mathrm{m} \mathrm{N \textrm {NH } _ { 3 }}$ feature (Figure 10) because of increasing noise toward longer wavelengths.

We also explore various conditions and atmospheric scenarios which could affect the detection level of ammonia in the atmosphere of our targets in the near-infrared: (1) varying concentration of ammonia in the atmosphere (Section 5.3) (2) varying atmospheric composition (Section 5.4); and (3) varying cloud decks (Section 5.5).

\subsection{Varying Concentration of Ammonia}

Using TOI-270 c observations with NIRISS and NIRSpec as an example, we vary the amount of ammonia in the atmosphere (Figure 11; Table 7). Following Seager et al. (2013b), the concentration of ammonia in the atmosphere is proportional to the biomass on the surface. A concentration of $11 \mathrm{ppm} \mathrm{NH}_{3}$ in the atmosphere of a temperature planet ${ }^{14}$ around a weakly active $\mathrm{M}$ dwarf is produced by a biomass density of $1 \mathrm{gm}^{-2}$. In

$\overline{{ }^{14} \text { A cold Haber world with } 90 \% \mathrm{H}_{2}: 10 \% \mathrm{~N}_{2}}$ with $T_{\text {eq }}=290 \mathrm{~K}$ and $1.75 R_{\oplus}$. 


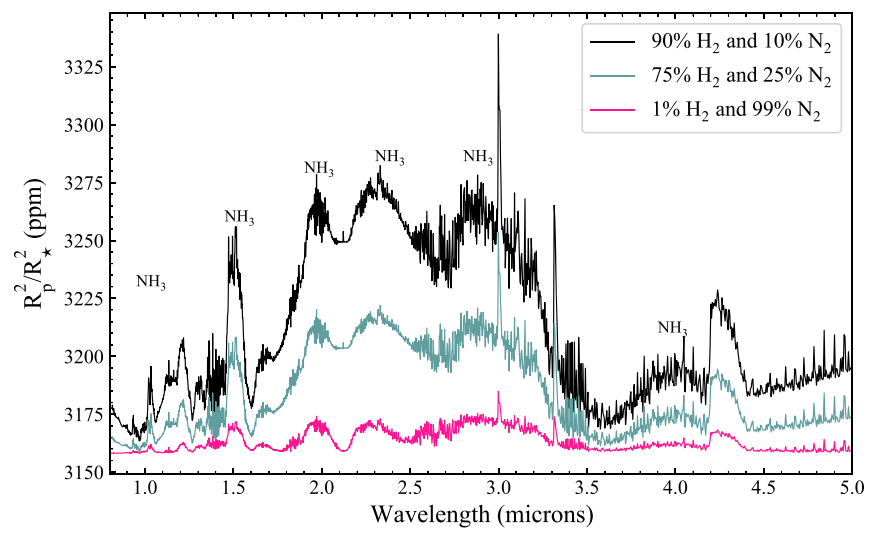

Figure 13. Modeled transmission spectra of TOI-270 c showing various atmospheric compositions compositions. The lines represent different hydrogen-dominated scenarios: a H-rich atmosphere $\left(90 \% \quad \mathrm{H}_{2}\right.$ and $\left.10 \% \mathrm{~N}_{2}\right)$, a $\mathrm{H}$-poor atmosphere $\left(1 \% \mathrm{H}_{2}\right.$ and $\left.99 \% \mathrm{~N}_{2}\right)$, and a $\mathrm{H}$-intermediate atmosphere $\left(75 \% \mathrm{H}_{2}\right.$ and $25 \% \mathrm{~N}_{2}$ ).

Table 7

Average $\mathrm{S} / \mathrm{N}$ and Transmission Signal Difference of Major $\mathrm{NH}_{3}$ Transmission Features for TOI-270 c Transmission Spectroscopy for Varying Concentrations of Ammonia

\begin{tabular}{|c|c|c|c|c|}
\hline $\begin{array}{l}\text { Concentration of } \\
\mathrm{NH}_{3}\end{array}$ & $\begin{array}{c}\text { Ammonia } \\
\text { Feature } \\
{[\mu \mathrm{m}]}\end{array}$ & $\begin{array}{l}\mathrm{S} / \mathrm{N} \\
{[\sigma]}\end{array}$ & $\begin{array}{c}<\mathrm{S} / \mathrm{N}> \\
{[\sigma]}\end{array}$ & $\begin{array}{c}\text { Transmission Sig- } \\
\text { nal Difference } \\
{[\mathrm{ppm}]}\end{array}$ \\
\hline \multirow[t]{4}{*}{$0.4 \mathrm{ppm}$} & 1.5 & 1.6 & 8.5 & 30 \\
\hline & 2.0 & 5.1 & & 22 \\
\hline & 2.3 & 5.2 & & 3 \\
\hline & 3.0 & 4.1 & & 36 \\
\hline \multirow[t]{4}{*}{$4.0 \mathrm{ppm}$} & 1.5 & 5.0 & 10.9 & 72 \\
\hline & 2.0 & 5.4 & & 35 \\
\hline & 2.3 & 5.1 & & 42 \\
\hline & 3.0 & 6.2 & & 92 \\
\hline \multirow[t]{4}{*}{40 ppm } & 1.5 & 9.2 & 16.0 & 142 \\
\hline & 2.0 & 7.2 & & 87 \\
\hline & 2.3 & 6.0 & & 91 \\
\hline & 3.0 & 9.0 & & 137 \\
\hline \multirow[t]{4}{*}{$400 \mathrm{ppm}$} & 1.5 & 11.9 & 19.5 & 231 \\
\hline & 2.0 & 8.8 & & 152 \\
\hline & 2.3 & 6.7 & & 106 \\
\hline & 3.0 & 10.5 & & 183 \\
\hline
\end{tabular}

contrast, a quiet M-type star would need less biomass- $1.4 \times$ $10^{-2} \mathrm{gm}^{-2}$, to produce the same $11 \mathrm{ppm}$ concentration of $\mathrm{NH}_{3}$ in the atmosphere (Seager et al. 2013a; Seager et al. 2013b).

We choose varying ammonia concentrations of $0.4,4.0,40$, and $400 \mathrm{ppm}$. A range of $1-10 \mathrm{ppm}$ concentration of $\mathrm{NH}_{3}$ is reasonable for cold Haber worlds. Our base simulations of detectability assume a $4.0 \mathrm{ppm}$ concentration of ammonia, so we explore the effects of a varying ammonia concentration on the transmission signal on TOI-270 c. Figure 12 shows that a higher concentration of ammonia produces higher signals for detection with the NIRSpec/G235M, NIRSpec/G395M, and NIRISS/SOSS modes (Table 7). In the calculation of $\mathrm{NH}_{3}$ signal as shown in Figure 12, we measure the transmission signal difference for the varying levels of ammonia in the atmosphere $(0.4,4.0,40$, and $400 \mathrm{ppm})$ for the $1.5,2.0,2.3$, and $3.0 \mu \mathrm{m}$ ammonia transmission features relative to the base transmission signal for a 0 -ppm ammonia mixing ratio.

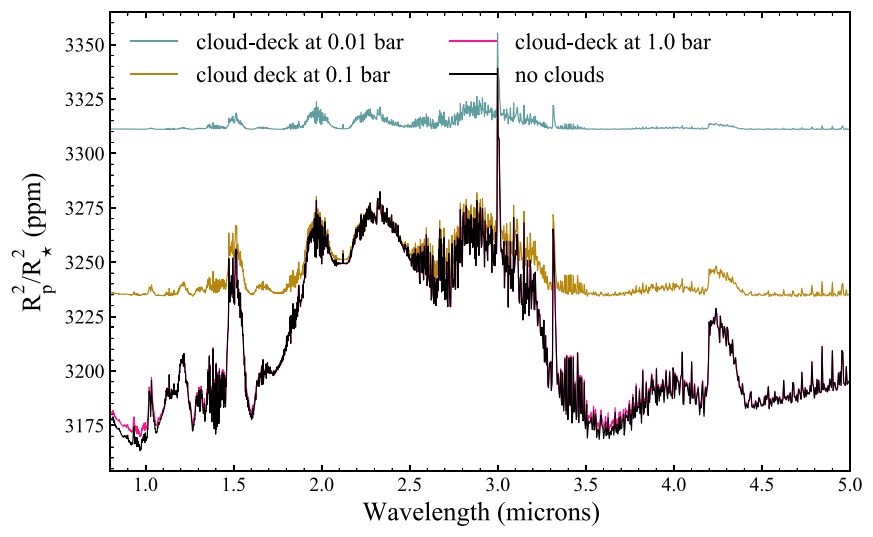

Figure 14. Transmission spectra of TOI-270 $\mathrm{c}$ shown with varying cloud deck structures at 0.01 bar (blue), 0.1 bar (gold), and 1 bar (pink). The varying ammonia feature are muted with a decreasing cloud deck pressure and are almost a flat line with a 0.01 bar cloud deck.

Table 8

Average $\mathrm{S} / \mathrm{N}$ of Major $\mathrm{NH}_{3}$ Transmission Features for TOI-270 c Transmission Spectroscopy for Different Atmospheric Compositions

\begin{tabular}{lccc}
\hline \hline TOI-270 c & $\begin{array}{c}\text { Ammonia Feature } \\
(\mu \mathrm{m})\end{array}$ & $\begin{array}{c}\mathrm{S} / \mathrm{N} \\
(\sigma)\end{array}$ & $\begin{array}{c}<\mathrm{S} / \mathrm{N}> \\
(\sigma)\end{array}$ \\
\hline H-rich & 1.5 & 5.0 & 10.9 \\
& 2.0 & 5.4 & \\
& 2.3 & 5.1 & \\
& 3.0 & 6.2 & 5.9 \\
\hline H-intermediate & 1.5 & 2.7 & \\
& 2.0 & 2.9 & 1.6 \\
& 2.3 & 2.7 & \\
\hline H-poor & 3.0 & 3.4 & \\
& 1.5 & 0.8 & \\
& 2.0 & 0.7 & \\
& 2.3 & 0.6 & \\
& 3.0 & 1.0 & \\
\end{tabular}

Table 9

Average $\mathrm{S} / \mathrm{N}$ of Major $\mathrm{NH}_{3}$ Transmission Features for TOI-270 c Transmission Spectroscopy for Varying Cloud Decks with a H-rich Atmosphere

\begin{tabular}{lccc}
\hline \hline TOI-270 c & $\begin{array}{c}\text { Ammonia Feature } \\
{[\mu \mathrm{m}]}\end{array}$ & $\begin{array}{c}\mathrm{S} / \mathrm{N} \\
{[\sigma]}\end{array}$ & $\begin{array}{c}<\mathrm{S} / \mathrm{N}> \\
{[\sigma]}\end{array}$ \\
\hline Cloud deck at 0.01 bar & 1.5 & 0.4 & 1.2 \\
& 2.0 & 0.3 & \\
& 2.3 & 0.3 & \\
& 3.0 & 1.0 & \\
\hline Cloud deck at 0.1 bar & 1.5 & 2.0 & \\
& 2.0 & 2.0 & 10.8 \\
& 2.3 & 2.2 & \\
\hline \multirow{2}{*}{ Cloud deck at 1.0 bar } & 3.0 & 3.2 & \\
& 1.5 & 4.9 & \\
& 2.0 & 5.3 & \\
& 2.3 & 5.0 & \\
& 3.0 & 6.1 & \\
\hline
\end{tabular}




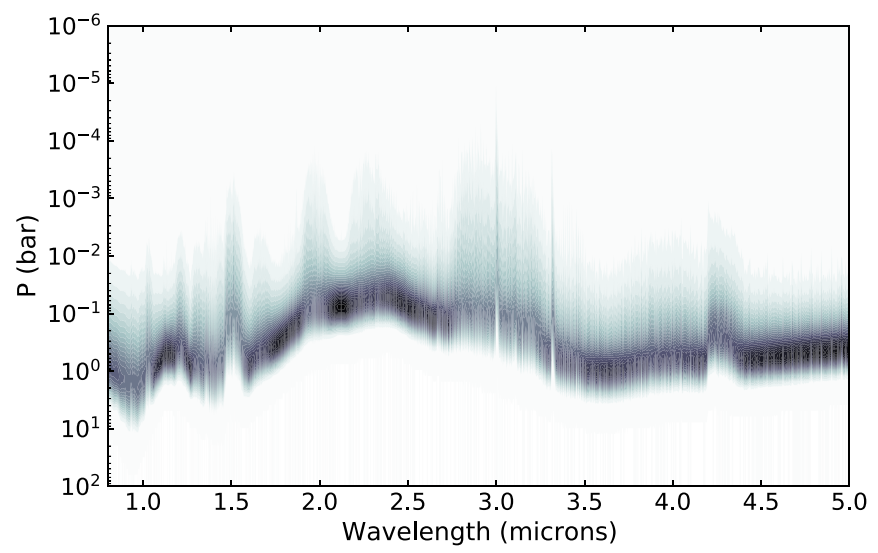

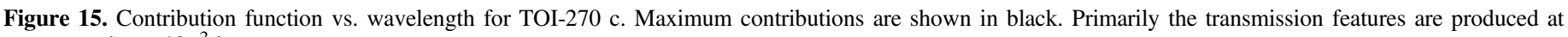
pressures above $10^{-2}$ bar.

Figure 3 in Seager et al. (2013b) shows that vertical mixing can only increase $\mathrm{NH}_{3}$ values higher up in the atmosphere, making it more detectable, so our simulation is a less optimistic case than the case that considers vertical mixing.

\subsection{Varying Atmospheric Composition}

Theoretical studies and observational works based on data from HST have explored the atmospheric compositions for gas dwarf atmospheres (e.g., Elkins-Tanton \& Seager 2008; MillerRicci et al. 2008; Seager \& Deming 2010; Benneke \& Seager 2012). Recently, HST observations showed that water vapor was present in the atmosphere, which indicates a thick hydrogen-rich gas envelope for K2-18 b (Benneke et al. 2019b; Madhusudhan et al. 2020), and evidence of a thick atmosphere for LHS 1140 b (Edwards et al. 2021).

To explore the diversity of gas dwarf atmospheres, we follow Chouqar et al. (2020) to consider the following cases: a hydrogen-rich atmosphere $\left(90 \% \mathrm{H}_{2}\right.$ and $\left.10 \% \mathrm{~N}_{2}\right)$, a hydrogenpoor atmosphere $\left(1 \% \mathrm{H}_{2}\right.$ and $\left.99 \% \mathrm{~N}_{2}\right),{ }^{15}$ and a hydrogenintermediate atmosphere $\left(75 \% \mathrm{H}_{2}\right.$ and $\left.25 \% \mathrm{~N}_{2}\right)$, in order to determine the effects on the detection of ammonia. Figure 13 shows that the strength of ammonia features corresponds to the amount of hydrogen present in the atmosphere. Compared to a hydrogen-rich and hydrogen-intermediate atmosphere, a hydrogen-poor atmosphere has a weaker transmission signal and $\mathrm{S} / \mathrm{N}$ -based on our transmission detection metric. A more quantitative summary is given in Table 8 .

\subsection{Cloud Decks}

One of the major challenges for the search of $\mathrm{NH}_{3}$ in gas dwarfs is the presence of clouds, which have been shown to mask transmission features (Helling 2019; Barstow 2021). Clouds have been shown to be present in the atmospheres of gas dwarfs (Kreidberg et al. 2014; Benneke et al. 2019b). For example, GJ 1214 b, has shown a flat transmission spectrum due to the effects of high-altitude clouds (Berta et al. 2012; Kreidberg et al. 2014).

We use petitRADTRANS to model the effect of varying levels of cloud deck structures in the atmospheres $(1,0.1$, and $0.01 \mathrm{bar}$ ). We find that a decreasing cloud deck pressure (i.e., increasing height of clouds) masks the $\mathrm{NH}_{3}$ atmospheric features to a near flat continuum and affects the detectability of

\footnotetext{
15 The hydrogen-poor atmosphere is constructed following the same method as used in Section 3.2 with $X_{H}=0.01$ and $X_{N}=0.99$.
}

major $\mathrm{NH}_{3}$ transmission feature (Figure 14). A more quantitative summary is given in Table $9 .^{16}$

We employ the use of a contribution function that indicates locations of a cloud deck layer in the atmosphere that transmission features are produced from and where spectral features begin to become muted.

We find that primarily the transmission features are produced at pressures higher than $10^{-2}$ bar in the atmosphere. Therefore, a cloud deck at the pressure level (and below) starts to mute the spectral features (Figure 15).

Hazes can impact spectra and produce flatter transmission spectra similar to clouds (Marley et al. 2013; Wunderlich et al. 2021). Our gray cloud treatment results in the same behavior as hazes in the near-infrared. Super Rayleigh slope due to hazes (Ohno \& Kawashima 2020) primarily impact the bluer optical wavelengths - so we do not explore the effects in this study.

\section{Atmospheric Retrieval Examples}

In previous sections, we provide an $\mathrm{S} / \mathrm{N}$-based metric to quantify the detectability of $\mathrm{NH}_{3}$. The metric guides us in prioritizing targets and determining conditions under which the detection is plausible. In this section, we provide a few examples on how $\mathrm{NH}_{3}$ can be detected and the abundance constrained under optimal conditions, e.g., a cloud-free atmosphere with low MMW for TOI-270 c, one of the most promising targets in our sample based on the $\mathrm{S} / \mathrm{N}$ metric.

A full exploration of parameter space will be conducted in a future paper to determine the threshold for detecting and constraining $\mathrm{NH}_{3}$ abundance. The purpose here is to (1) demonstrate that $\mathrm{NH}_{3}$ and $\mathrm{H}_{2} \mathrm{O}$ abundance can be retrieved independently despite their overlapping wavelengths in absorption; and (2) understand the impact of cloud on the retrieval precision.

\subsection{Setting Up the Retrieval}

We use the simulated JWST data for TOI-270 $\mathrm{c}$ as the input. To model the simulated data, we use PETITRADTRANS (Mollière et al. 2019) with the following free parameters: surface gravity, planet radius, temperature for the isothermal atmosphere, cloud deck pressure, and mass mixing ratios for different species that are being considered. In a Bayesian

\footnotetext{
${ }^{16}$ The $\mathrm{S} / \mathrm{N}[\sigma]$ values are calculated using our transmission detection metric (Equation (4))
} 

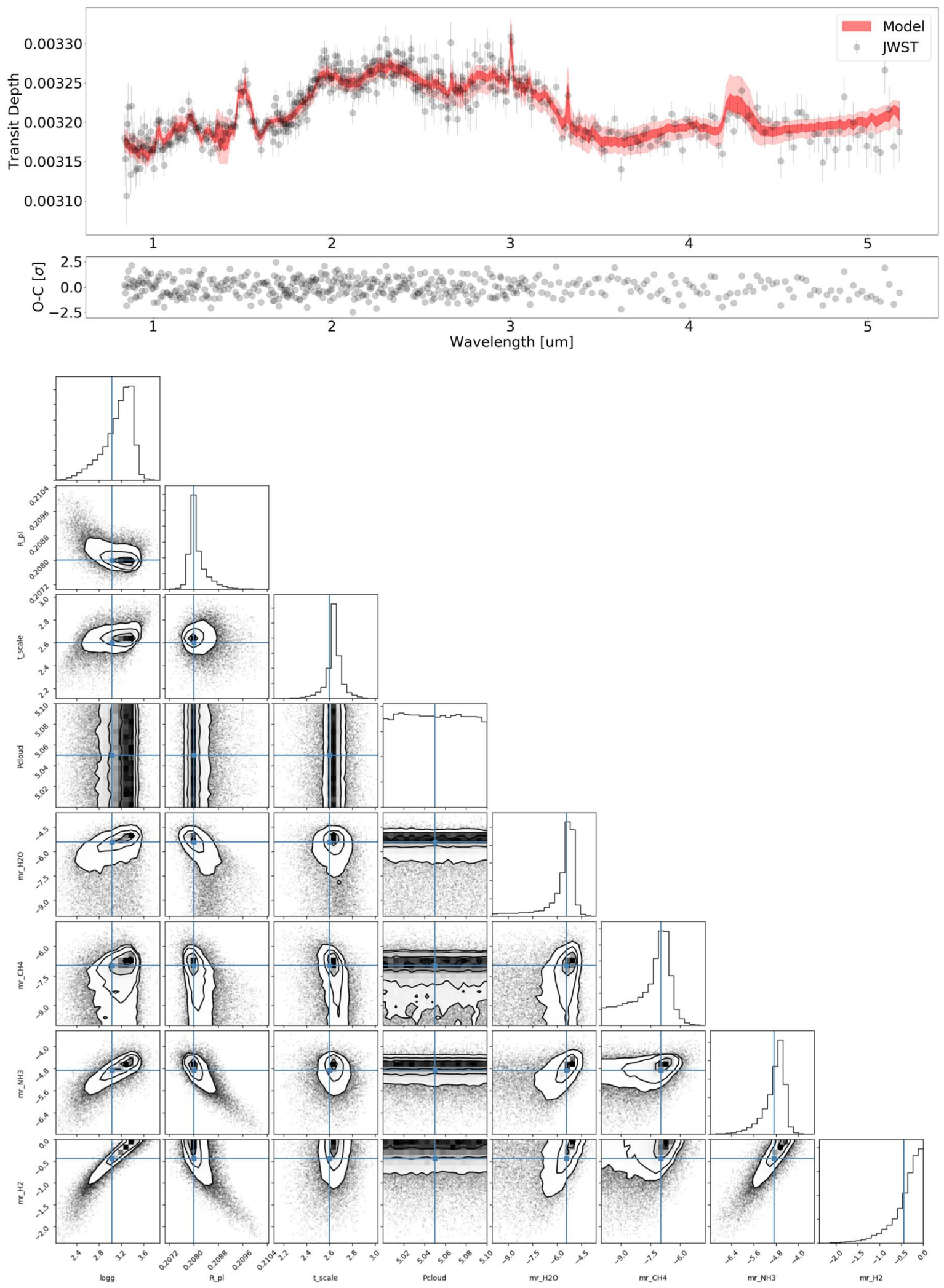

Figure 16. Top: simulated JWST data vs. the retrieved model, and the $\mathrm{O}-\mathrm{C}$ plot that shows the residual. Bottom: corner plot for the parameters that are used in the retrieval along with true values that are used in generating the JWST data. Contours are shown at $0.5 \sigma, 1 \sigma, 1.5 \sigma$, and $2 \sigma$.

framework, we use PYMULTINEST (Buchner et al. 2014) to sample the posteriors. The priors are listed in Table 10. The likelihood function is $\sum \exp \left[-(\mathcal{D}-\mathcal{M})^{2} / \mathcal{E}^{2}\right] / 2$, where $\mathcal{D}$ is data, $\mathcal{M}$ is model, and $\mathcal{E}$ is the error term. The input values of the retrieval tests can be found in Table 10. For PyMultiNest, we use 2000 live points. 
Table 10

Parameters used in Retrieval, Their Priors, Input, and Retrieved Values

\begin{tabular}{|c|c|c|c|c|c|c|c|}
\hline \multirow{2}{*}{ Parameter } & \multirow[t]{2}{*}{ Unit } & \multirow{2}{*}{ Type } & \multirow{2}{*}{ Lower } & \multirow{2}{*}{ Upper } & \multirow{2}{*}{ Input } & \multicolumn{2}{|c|}{ Retrieved } \\
\hline & & & & & & Fixed & Free \\
\hline$\overline{\text { Surface gravity (logg) }}$ & cgs & Uniform & 2.0 & 5.0 & 3.0395 & $3.20_{-0.34}^{+0.19}$ & $3.12_{-0.35}^{+0.24}$ \\
\hline Planet radius $\left(R_{P}\right)$ & $R_{\text {Jupiter }}$ & Uniform & 0.2 & 0.5 & 0.208 & $0.20802_{-0.00013}^{+0.00035}$ & $0.20805_{-0.00017}^{+0.00035}$ \\
\hline Temperature $\left.\left(T_{\text {iso }}\right)\right)$ & K & Log-uniform & 10 & 3300 & 400 & $436_{-47}^{+53}$ & $436_{-38}^{+53}$ \\
\hline Cloud pressure $\left(\log \left(P_{\text {cloud }}\right)\right)$ & bar & Log-uniform & -6 & 6 & 5.05 & fixed & $2.46_{-2.18}^{+2.38}$ \\
\hline $\mathrm{H}_{2} \mathrm{O}$ mixing ratio $\left(\log \left(\mathrm{mr}_{\mathrm{H}_{2} \mathrm{O}}\right)\right)$ & $\cdots$ & Log-uniform & -10 & 0 & -5.44 & $-5.39_{-0.94}^{+0.31}$ & $-5.67_{-0.99}^{+0.44}$ \\
\hline $\mathrm{CO}$ mixing ratio $\left(\log \left(\mathrm{m} R_{\mathrm{CO}}\right)\right)$ & $\cdots$ & Log-uniform & -10 & 0 & -8.25 & fixed & $-8.35_{-1.09}^{+1.33}$ \\
\hline $\mathrm{CO}_{2}$ mixing ratio $\left(\log \left(\mathrm{mr}_{\mathrm{CO}_{2}}\right)\right)$ & $\cdots$ & Log-uniform & -10 & 0 & -7.55 & fixed & $-8.14_{-0.99}^{+0.66}$ \\
\hline $\mathrm{CH}_{4}$ mixing ratio $\left(\log \left(\mathrm{mr}_{\mathrm{CH}_{4}}\right)\right)$ & $\cdots$ & Log-uniform & -10 & 0 & -6.99 & $-7.20_{-1.54}^{+0.56}$ & $-7.34_{-1.31}^{+0.62}$ \\
\hline OH mixing ratio $\left(\log \left(\mathrm{mr}_{\mathrm{OH}}\right)\right)$ & $\ldots$ & Log-uniform & -10 & 0 & -14.47 & fixed & $-6.11_{-2.38}^{+1.29}$ \\
\hline $\mathrm{NH}_{3}$ mixing ratio $\left(\log \left(\mathrm{mr}_{\mathrm{NH}_{3}}\right)\right)$ & $\cdots$ & Log-uniform & -10 & 0 & -4.86 & $-4.76_{-0.46}^{+0.23}$ & $-4.89_{-0.51}^{+0.31}$ \\
\hline $\mathrm{H}_{2}$ mixing ratio $\left(\log \left(\mathrm{mr}_{\mathrm{H}_{2}}\right)\right)$ & $\cdots$ & Log-uniform & -10 & 0 & -0.44 & $-0.29_{-0.42}^{+0.20}$ & $-0.37_{-0.44}^{+0.26}$ \\
\hline $\mathrm{HCN}$ mixing ratio $\left(\log \left(\mathrm{mr}_{\mathrm{HCN}}\right)\right)$ & $\ldots$ & Log-uniform & -10 & 0 & -8.27 & fixed & $-8.05_{-1.28}^{+1.38}$ \\
\hline Wavelength shift $\left(\Delta_{\lambda}\right)$ & $\mu \mathrm{m}$ & Uniform & -0.1 & 0.1 & 0.0 & fixed & $0.00066_{-0.00281}^{+0.00198}$ \\
\hline
\end{tabular}

\subsection{Fixing Cloud Deck and Other Minor Species}

In this case, we use the simulated data for the cloud-free lowMMW case for TOI-270 c (Figure 8). In the retrieval, we assume the cloud deck at $10^{5}$ bar, which is well below the pressure range that contributes to the absorption (i.e., $10^{-3}-10$ bar). This is therefore an a priori cloudy-free case for the retrieval. Furthermore, we fix the abundance for species whose mass mixing ratios are below $5 \times 10^{-8}$ for the following reasons. First, these species have low abundances and may not be practically detected given the JWST data quality. This will be shown in the next example. Second, we want to focus on $\mathrm{NH}_{3}$ and $\mathrm{H}_{2} \mathrm{O}$ in this example and check if the two species can be reasonably measured despite overlapping absorption wavelengths regions.

As shown in Figure 16, $\mathrm{NH}_{3}$ and $\mathrm{H}_{2} \mathrm{O}$ can be detected in our retrieval, and their abundances are within $1 \sigma$ from the input values. The comparison between simulated data points and retrieved spectra shows a good agreement, although the $2 \sigma$ region for the modeled spectra does not cover the majority of the data points. This is particularly the case for the 2.0 and $2.3 \mathrm{NH}_{3}$ features. We attribute this issue to the limitation of our modeling software in generating arbitrary shapes of spectra, but point out that the limitation of modeling spectra can be properly accounted for by adding a Gaussian process component in the retrieval process (e.g., Wang et al. 2020).

We have two ways of quantifying the detection significance. First, given the $\sim 50,000$ posterior samples and that no point falls into the $\left[10^{-10}\right.$ to $\left.10^{-9}\right]$ mixing ratio bin, we have a lower limit of $4.2 \sigma$ detection for $\mathrm{NH}_{3}$ and $\mathrm{H}_{2} \mathrm{O}$. Second, using the retrieved $\mathrm{NH}_{3}$ abundance of $-4.76 \mathrm{dex}$ and a $1 \sigma$ error bar of 0.46 dex (see Table 10), the distance to the lower edge of the prior -10 dex is $11.4 \sigma$. This is consistent with values reported in Table 6: the quadrature summation of $\mathrm{S} / \mathrm{N}$ for the 1.5, 2.0, 2.3 , and $3.0 \mathrm{NH}_{3}$ features is $10.9 \sigma$.

\subsection{Cloud Deck as a Free Parameter}

We then run a retrieval analysis on the full parameter set that includes (1) the cloud deck pressure; and (2) all minor species with mass mixing ratio lower than $5 \times 10^{-8}$. This is to demonstrate that the retrieval works with the full parameter set and returns a reasonable result when compared to the input parameters.
We have an upper limit for the cloud deck pressure at $\sim 3$ bar. This is consistent with the contribution function (Figure 15) which shows that the majority of planet transmission signal comes from atmospheric layers with pressures lower than 3 bar. Note that the prior for the cloud deck pressure covers a range from $10^{-6}$ to $10^{6}$ bar. The comparison between the data and the retrieved modeled spectra is similar to Figure 16 and we therefore do not include a comparison figure for this case. The corner plot is shown in Figure 17.

\section{Discussion}

\subsection{Comparing to Chouqar et al. (2020)}

Chouqar et al. (2020) performed a comprehensive study on the properties of the TOI-270 system including the detectability of an atmosphere and individual molecules using the NIRISS (SOSS), and NIRSpec/G395M modes for transmission spectroscopy in the near-infrared.

To calculate the total expected $\mathrm{S} / \mathrm{N}$ and number of transits to detect spectral features so that a $<\mathrm{S} / \mathrm{N}\rangle \geqslant 5$, the approach presented in Lustig-Yaeger et al. (2019) is utilized. Similar to Lustig-Yaeger et al. (2019), an S/N scaling relation is developed by running the PandExo JWST noise model across a grid with transits ranging from 1-100. An S/ N is determined based on the difference between the model spectrum and featureless fiducial spectrum. This is the basis of the approach used, but a more detailed description of the approach is available in Lustig-Yaeger et al. (2019) and Chouqar et al.(2020).

We find that for both instrument modes there are discrepancies between our results for TOI-270 c, also seen for TOI-270 d. Similarly to our work, Chouqar et al. (2020) utilize petitRADTRANS to generate the spectra, for a clear hydrogen-rich atmosphere; however, they used an MMW $=2.39\left(\mathrm{X}_{H}=0.9\right)$, as opposed to our hydrogen-rich atmosphere with an $\mathrm{MMW}=4.55$. As a result, their simulated $2.0 \mu \mathrm{m} \mathrm{NH}_{3}$ feature for TOI- $270 \mathrm{c}$ has an $\sim 200 \mathrm{ppm}$ signal from the baseline and our spectrum of TOI$270 \mathrm{c}$ has an $\sim 100$ ppm signal.

For TOI-270 c with a H-rich atmosphere, they find that the NIRISS (SOSS) instrument requires only one transit to detect ammonia ${ }^{17}$ with an $\mathrm{S} / \mathrm{N}=18$. We attempt to replicate their results using their MMW value, $R \sim 10$, and the number of

\footnotetext{
$\overline{{ }^{17} \text { Chouqar et al. (2020) consider the } 2.0 \mu \mathrm{m}}$ ammonia feature.
} 


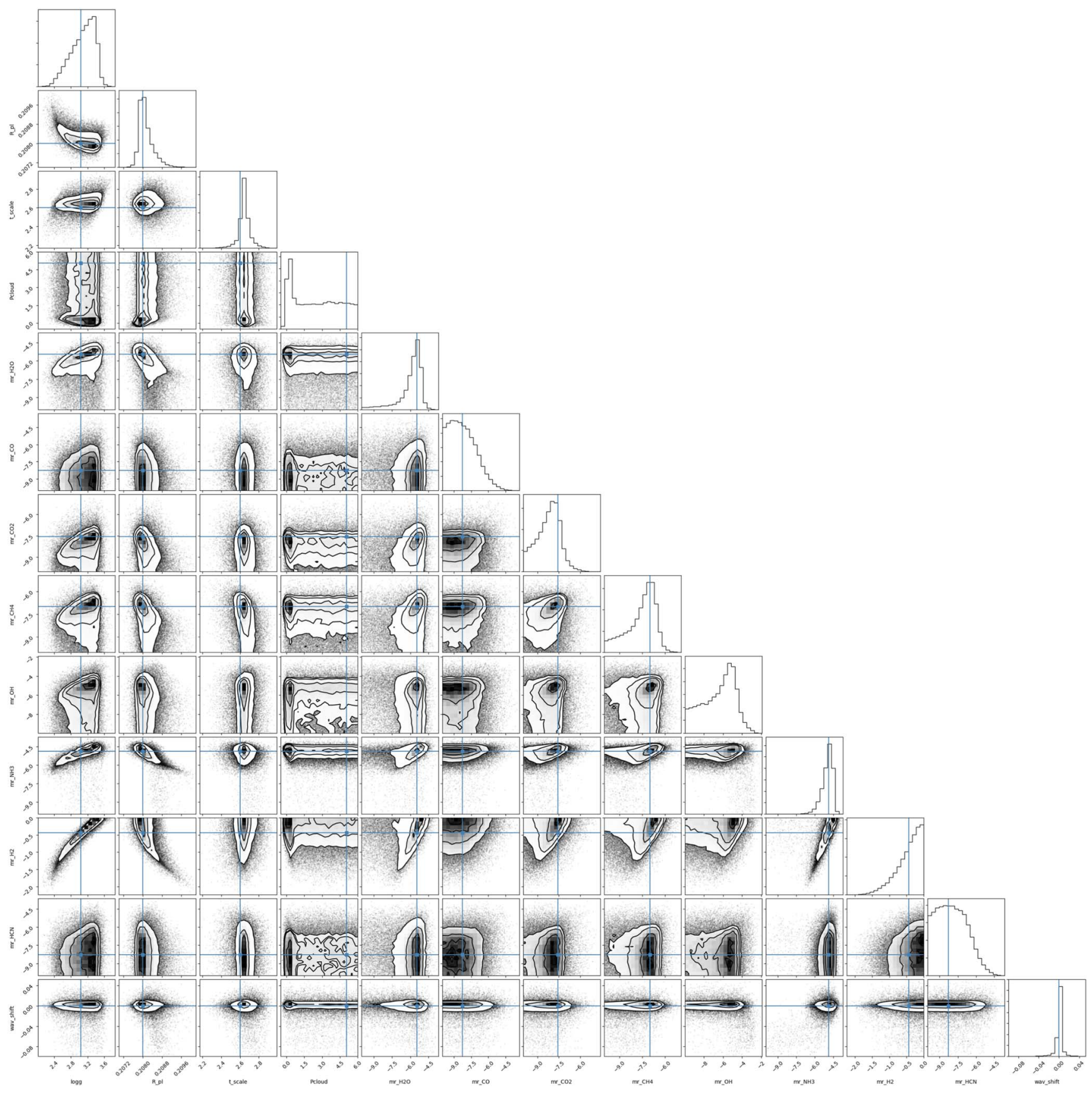

Figure 17. Corner plot for the full parameter set that is used in the retrieval along with true values that are used in generating the JWST data.

transits set to 1 . For the same $2.0 \mu \mathrm{m}$ feature using NIRISS (SOSS) we find an $\mathrm{S} / \mathrm{N}=12.5$.

They also find that with NIRSpec/G395M for one transit, the ammonia feature (we assume they are referring to the 3.0 $\mu \mathrm{m}$ feature, based on their Figure 5) has an $\mathrm{S} / \mathrm{N}$ of 5.0. For NIRSpec/G395M we find an $\mathrm{S} / \mathrm{N}=4.0$ for the $3.0 \mu \mathrm{m}$ feature. Therefore, our NIRSpec/G395M simulation is roughly consistent with the result in Chouqar et al. (2020).

\subsection{Comparing to Wunderlich et al. (2021)}

Wunderlich et al. (2021) performed an assessment of the detectability of biosignatures for LHS $1140 \mathrm{~b}$ with NIRSpec/
PRISM. For the purpose of comparison, we look specifically at the $3.0 \mu \mathrm{m} \mathrm{NH}_{3}$ feature they considered for their study.

In their study, an $\mathrm{S} / \mathrm{N}=5$ is utilized to determine whether or not a spectral feature is detectable. The method employed involves the subtraction of the full transmission spectra with all included absorption species from the spectrum excluding contribution from individual species. The $\mathrm{S} / \mathrm{N}$ determination is based on Wunderlich et al. (2019).

Differing from petitRADTRANS, which is based on a radiative transfer model, the atmosphere of LHS $1140 \mathrm{~b}$ from Wunderlich et al. (2021) is built using the radiative-convective photochemistry-climate coupled model, 1D-TERRA. The 1DTERRA model has inputs of the following: $\mathrm{P}-\mathrm{T}$ profiles, initial 
compositions, stellar spectrum, and ion-pair production rates. Detailed schematics of the full model description is shown in Figure 1 of Scheucher et al. (2020).

Compared to our study, Wunderlich et al. (2021) additionally varies the concentration of $\mathrm{CH}_{4}$ with the low $\mathrm{CH}_{4}$ scenario assuming a VMR of $\sim 1 \times 10^{-6}$, which is about two orders of magnitude higher than our VMR for $\mathrm{CH}_{4}$ of $\sim 2.9 \times 10^{-8}$ for our low-MMW $90 \% \mathrm{H}_{2}$ model atmosphere.

They find that with NIRSpec/PRISM - to detect the $3.0 \mu \mathrm{m}$ $\mathrm{NH}_{3}$, the required time for a $\mathrm{H}_{2}$-dominated atmosphere with a low $\mathrm{CH}_{4}$ scenario, the minimum number of transits would be $\sim 30$ transits, to achieve an $\mathrm{S} / \mathrm{N}$ of 5 . Overall, Wunderlich et al. (2021) find that to detect $\mathrm{NH}_{3}$ the required time would be between $10-50$ transits $(\sim 40-200) \mathrm{hr}$ of observing time assuming non-cloudy conditions. to Wunderlich et al. (2021) we do not consider the NIRSpec/PRISM as this mode saturates the detector for LHS 1140 b (Table 5; Wunderlich et al. 2021); instead we employed the use of NIRSpec/G235M, NIRSpec/ G395M, and NIRISS (SOSS) modes. For LHS $1140 \mathrm{~b}$ based on our defined detection metric, we find that using NIRISS (SOSS) for the $3.0 \mu \mathrm{m} \mathrm{NH}_{3}$, with 10 transits we would find a $3.1 \sigma$ detection given clear atmosphere conditions in a H-rich atmosphere, which corresponds to $\sim 60 \mathrm{hr}$ of observing time. For 30 transits $(\sim 180)$ hr of observing time) we find an $\mathrm{S} / \mathrm{N} \sim$ $4.4 \sigma$. This is qualitatively consistent with their conclusion that $\mathrm{NH}_{3}$ can be detected in 10-50 transits, and the detection of $\mathrm{NH}_{3}$ can be made in the presence of higher concentration of $\mathrm{CH}_{4}$.

\subsection{False Positives for Biotic $\mathrm{NH}_{3}$}

Ammonia has been proposed as a biosignature in hydrogendominated atmospheres; however, it is not immune to false positives (Seager et al. 2013b; Catling et al. 2018). Seager et al. (2013b) defined three major factors that can cause ammonia to be produced abiotically in these atmospheres: (1) a rocky world with a surface temperature of $\sim 820 \mathrm{~K}^{18}$ (2) the natural production of $\mathrm{NH}_{3}$ in the atmospheres of mini-Neptunes, and (3) planets that have outgassed $\mathrm{NH}_{3}$ during evolution. Seager et al. (2013b) notes that targets have to be evaluated on a caseby-case basis. Additionally, according to the thesis work by Evan Sneed, ${ }^{19}$ another cause of false positives for $\mathrm{NH}_{3}$ include comet collisions that contain inorganic ammonia ice.

\subsection{Other Factors in Prioritizing Targets}

Other factors beyond the $\mathrm{S} / \mathrm{N}$ of $\mathrm{NH}_{3}$ for our targets should be considered when prioritizing targets for JWST time. These factors include precise mass and radius measurements.

For example, only K2-18 b (Benneke et al. 2019b) and LHS 1140 b (Dittmann et al. 2017; Lillo-Box et al. 2020) have better than $\sim 15 \%$ and $\sim 3 \%$ precision in mass and radius measurements. This allows for a proper modeling of the planet interior (Lillo-Box et al. 2020) and atmosphere composition (Madhusudhan et al. 2020), which is essential to interpret the results of detection and non-detection.

\footnotetext{
18 At high surface temperatures, ammonia can be produced by the traditional Haber process from an iron surface.

19 https://zenodo.org/record/4015708\#.YJXiGy1h124
}

\section{Conclusion}

We modeled seven promising gas dwarfs for the detection of the potential biosignature ammonia using the MIRI, NIRSpec, and NIRISS instruments on the upcoming JWST mission: GJ 143 b, TOI-270 c, TOI-270 d, K2-18 b, K2-3 c, LHS 1140 b, and LP 791-18 c.

MIRI LRS has a systemic noise limit of $\sim 12.6 \mathrm{ppm}$ for 10 eclipses, where the $10.3-10.8 \mu \mathrm{m} \mathrm{NH}_{3}$ feature for the majority of our targets is not detectable due to the limitation by this systematic noise limit.

The most promising targets for emission spectroscopy with MIRI LRS is LP 791-18 c in terms of expected emission spectroscopy signal (Figure 3). However, in practice, even with 10 transits (i.e $\sim 40 \mathrm{hr}$ of observing time) we cannot realistically detect the signal (Figure 7) due to large photon noise.

We defined a detection metric for transmission spectroscopy and utilize the following modes to perform JWST simulations using a baseline of 10 transits for non-cloudy and low-MMW atmospheres: NIRSpec/G395M, NIRSpec/G235M, NIRISS (SOSS), and MIRI LRS. We compile a ranking list for observing targets with JWST based on the $\mathrm{S} / \mathrm{N}$ detection metric of six major $\mathrm{NH}_{3}$ features $(1.5,2.0,2.3,3.0,6.1$, and 103-10.8 $\mu \mathrm{m})$. The rank list follows as such: TOI-270 c, LP 79118 c TOI-270 d, LHS 1140 b, K2-3 c, K2-18 b, and GJ 143 b. TOI-270 c, is ranked first as it has the highest average $S / N$ and GJ $143 \mathrm{~b}$ is ranked last as the host star saturates the majority of the chosen observing modes.

We also test the capabilities of transmission spectroscopy with MIRI LRS to detect the 6.1 and 10.3-10.8 $\mu \mathrm{m}$ feature, and overall we find that the $6.1 \mu \mathrm{m}$ feature is more suitable for detection than the $10.3-10.8 \mu \mathrm{m} \mathrm{NH}$ feature for transmission spectroscopy with MIRI LRS.

Using TOI-270 c as an example, we test a variety of scenarios to determine the effect of detectability of ammonia: varying concentration of ammonia (Section 5.3), varying atmospheric composition (Section 5.4), and including effects of cloud decks (Section 5.5).

For a baseline of 10 transits, we find that a higher concentration of ammonia (400 ppm) in the atmospheres produces a higher transmission signal difference. Similarly, we model the effects of a varying hydrogen composition for TOI-270 c, and find that a H-rich $(90 \%$ hydrogen based atmosphere) produces a higher averaged $\mathrm{S} / \mathrm{N}$ detection, about a factor of 10 , compared to a $\mathrm{H}$-poor (1\% hydrogen based atmosphere). Lastly, in the presence of cloud decks, the average $\mathrm{S} / \mathrm{N}$ detection of ammonia decreases to $1.2 \sigma$ and $4.8 \sigma$ from $10.9 \sigma$ from the cloud deck of 0.01 and 0.1 bar, respectively.

We provide examples of atmospheric retrieval (Section 6) and show that $\mathrm{NH}_{3}$ and $\mathrm{H}_{2} \mathrm{O}$ can be detected in amenable conditions, i.e., a cloud-free atmosphere with a low MMW. For example, based on the posterior distribution of $\mathrm{NH}_{3}$ in Figure 16 , the detection significance is $\sim 11 \sigma$, which is roughly consistent with the $\mathrm{S} / \mathrm{N}$ value that is reported in Table 6 for the four $\mathrm{NH}_{3}$ feature from 1.5-3.0 $\mu \mathrm{m}$. The comparison provides corroborative evidence for the validity of our method of calculating $\mathrm{S} / \mathrm{N}$ and the retrieval code. The retrieval can also constrain the pressure level of a cloud deck.

This work demonstrates that JWST will provide unprecedented wavelength coverage and light collecting area for atmospheric studies of gas dwarfs and their potential biosignatures. 
This work benefited from involvement in ExoExplorers, which is sponsored by the Exoplanets Program Analysis Group (ExoPAG) and NASAs Exoplanet Exploration Program Office (ExEP). The authors also wish to thank Victoria Meadows and Natasha Batalha for their helpful advice and suggestions for this manuscript. C. P. thanks the LSSTC Data Science Fellowship Program, which is funded by LSSTC, NSF Cybertraining Grant \#1829740, the Brinson Foundation, and the Moore Foundation; her participation in the program has benefited this work. This work has made use of data from the European Space Agency (ESA) mission Gaia (https://www. cosmos.esa.int/gaia), processed by the Gaia Data Processing and Analysis Consortium (DPAC, https://www.cosmos.esa. int/web/gaia/dpac/consortium). Funding for the DPAC has been provided by national institutions, in particular the institutions participating in the Gaia Multilateral Agreement

We thank the anonymous referee for their time providing helpful comments that improved the quality of this paper. This research has made use of the NASA Exoplanet Archive, which is operated by the California Institute of Technology, under contract with the National Aeronautics and Space Administration under the Exoplanet Exploration Program. This project is supported, in part, by funding from Two Sigma Investments, LP. Any opinions, findings, and conclusions or recommendations expressed in this material are those of the authors and do not necessarily reflect the views of Two Sigma Investments, LP.

NASA's Astrophysics Data System Bibliographic Services together with the VizieR catalog access tool and SIMBAD database operated at CDS, Strasbourg, France, were invaluable resources for this work. This publication makes use of data products from the Two Micron All Sky Survey, which is a joint project of the University of Massachusetts and the Infrared Processing and Analysis Center/California Institute of Technology, funded by the National Aeronautics and Space Administration and the National Science Foundation.

Facility: Exoplanet Archive.

Software: Pandexo (Batalha et al. 2017), petitRADTRANS (Mollière et al. 2019).

\section{ORCID iDs}

Caprice L. Phillips (iD https://orcid.org/0000-0001-5610-5328 Ji Wang (1) https://orcid.org/0000-0002-4361-8885

Sarah Kendrew (i) https://orcid.org/0000-0002-7612-0469

Thomas P. Greene (iD https://orcid.org/0000-0002-8963-8056

Renyu Hu (D) https://orcid.org/0000-0003-2215-8485

Wendy R. Panero (iD https://orcid.org/0000-0001-5753-2532

Joseph Schulze (iD https://orcid.org/0000-0003-3570-422X

\section{References}

Akins, A. B., Lincowski, A. P., Meadows, V. S., \& Steffes, P. G. 2021, ApJL, 907, L27

Almenara, J. M., Astudillo-Defru, N., Bonfils, X., et al. 2015, A\&A, 581, L7 Barstow, J. K. 2021, A\&G, 62, 1.36

Batalha, N. E., Lewis, N. K., Line, M. R., Valenti, J., \& Stevenson, K. 2018, ApJL, 856, L34

Batalha, N. E., Mandell, A., Pontoppidan, K., et al. 2017, PASP, 129, 064501 Beichman, C., Benneke, B., Knutson, H., et al. 2014, PASP, 126, 1134 Benneke, B., Knutson, H. A., Lothringer, J., et al. 2019a, NatAs, 3, 813 Benneke, B., \& Seager, S. 2012, ApJ, 753, 100

Benneke, B., Werner, M., Petigura, E., et al. 2017, ApJ, 834, 187

Benneke, B., Wong, I., Piaulet, C., et al. 2019b, ApJL, 887, L14

Berta, Z. K., Charbonneau, D., Désert, J.-M., et al. 2012, ApJ, 747, 35

Borucki, W. J., Koch, D., Basri, G., et al. 2010, Sci, 327, 977

Buchhave, L. A., Bizzarro, M., Latham, D. W., et al. 2014, Natur, 509, 593
Buchner, J., Georgakakis, A., Nandra, K., et al. 2014, A\&A, 564, A125 Catling, D. C., Krissansen-Totton, J., Kiang, N. Y., et al. 2018, AsBio, 18, 709 Chaplin, M. F. 2019, Encyclopedia of Water (New York: Wiley)

Chouqar, J., Benkhaldoun, Z., Jabiri, A., et al. 2020, MNRAS, 495, 962

Crossfield, I. J. M., Ciardi, D. R., Petigura, E. A., et al. 2016, ApJS, 226, 7 Crossfield, I. J. M., Waalkes, W., Newton, E. R., et al. 2019, ApJL, 883, L16 Cutri, R. M., Skrutskie, M. F., van Dyk, S., et al. 2003, yCat, 2246, 0 Deming, D., Bouwman, J., Dicken, D., et al. 2021, A Time Series Calibration of Medium Resolution Spectroscopy with MIRI, JWST Proposal. Cycle 1, ID. \#1556

Des Marais, D. J., Harwit, M. O., Jucks, K. W., et al. 2002, AsBio, 2, 153 Dittmann, J. A., Irwin, J. M., Charbonneau, D., et al. 2017, Natur, 544, 333 Dragomir, D., Teske, J., Günther, M. N., et al. 2019, ApJL, 875, L7

Edwards, B., Changeat, Q., Mori, M., et al. 2021, AJ, 161, 44

Elkins-Tanton, L. T., \& Seager, S. 2008, ApJ, 685, 1237

Fortenbach, C. D., \& Dressing, C. D. 2020, PASP, 132, 054501

Fressin, F., Torres, G., Charbonneau, D., et al. 2013, ApJ, 766, 81

Fulton, B. J., Petigura, E. A., Howard, A. W., et al. 2017, AJ, 154, 109

Gaia Collaboration, Brown, A. G. A., Vallenari, A., et al. 2018, A\&A, 616, A1

Gillon, M., Demory, B.-O., Van Grootel, V., et al. 2017, NatAs, 1, 0056

Glasse, A., Rieke, G. H., Bauwens, E., et al. 2015, PASP, 127, 686

Greaves, J. S., Bains, W., Petkowski, J. J., et al. 2020, arXiv:2012.05844

Greaves, J. S., Richards, A. M. S., Bains, W., et al. 2021, NatAs, 5, 655

Greene, T. P., Line, M. R., Montero, C., et al. 2016, ApJ, 817, 17

Günther, M. N., Pozuelos, F. J., Dittmann, J. A., et al. 2019, NatAs, 3, 1099

Helling, C. 2019, AREPS, 47, 583

Hu, R. 2021, ApJ, 921, 27

Husser, T. O., Wende-von Berg, S., Dreizler, S., et al. 2013, A\&A, 553, A6

Kendrew, S., Dicken, D., Bouwman, J., et al. 2018, Proc. SPIE, 10698, $106983 \mathrm{U}$

Kendrew, S., Scheithauer, S., Bouchet, P., et al. 2015, PASP, 127, 623

Kosiarek, M. R., Crossfield, I. J. M., Hardegree-Ullman, K. K., et al. 2019, AJ, 157, 97

Kreidberg, L., Bean, J. L., Désert, J.-M., et al. 2014, Natur, 505, 69

Lillo-Box, J., Figueira, P., Leleu, A., et al. 2020, A\&A, 642, A121

Lincowski, A. P., Meadows, V. S., Crisp, D., et al. 2021, ApJL, 908, L44

Lustig-Yaeger, J., Meadows, V. S., \& Lincowski, A. P. 2019, AJ, 158, 27

Madhusudhan, N., Nixon, M. C., Welbanks, L., Piette, A. A. A., \& Booth, R. A. 2020, ApJL, 891, L7

Madhusudhan, N., Piette, A. A. A., \& Constantinou, S. 2021, ApJ, 918, 1

Marley, M. S., Ackerman, A. S., Cuzzi, J. N., \& Kitzmann, D. 2013, in Clouds and Hazes in Exoplanet Atmospheres, ed. S. J. Mackwell et al. (Tuscon, AZ: Univ. Arizona Press), 367

Ment, K., Dittmann, J. A., Astudillo-Defru, N., et al. 2019, AJ, 157, 32

Miller-Ricci, E., Seager, S., \& Sasselov, D. 2008, ApJ, 690, 1056

Mollière, P., Wardenier, J. P., van Boekel, R., et al. 2019, A\&A, 627, 67

Mousis, O., Deleuil, M., Aguichine, A., et al. 2020, ApJL, 896, L22

Nixon, M. C., \& Madhusudhan, N. 2021, MNRAS, 505, 3414

Ohno, K., \& Kawashima, Y. 2020, ApJL, 895, L47

Prinn, R. G., \& Olaguer, E. P. 1981, JGR, 86, 9895

Ricker, G. R., Winn, J. N., Vanderspek, R., et al. 2015, JATIS, 1, 014003

Rocchetto, M., Waldmann, I. P., Venot, O., Lagage, P. O., \& Tinetti, G. 2016, ApJ, 833, 120

Rogers, L. A. 2015, ApJ, 801, 41

Sarkis, P., Henning, T., Kürster, M., et al. 2018, AJ, 155, 257

Scheucher, M., Wunderlich, F., Grenfell, J. L., et al. 2020, ApJ, 898, 44

Seager, S., Bains, W., \& Hu, R. 2013a, ApJ, 777, 95

Seager, S., Bains, W., \& Hu, R. 2013b, ApJ, 775, 104

Seager, S., \& Deming, D. 2010, ARA\&A, 48, 631

Seager, S., Petkowski, J. J., Gao, P., et al. 2021, AsBio, 21, 1206

Sinukoff, E., Howard, A. W., Petigura, E. A., et al. 2016, ApJ, 827, 78

Snellen, I. A. G., Désert, J. M., Waters, L. B. F. M., et al. 2017, AJ, 154, 77

Snellen, I. A. G., Guzman-Ramirez, L., Hogerheijde, M. R., Hygate, A. P. S., \& van der Tak, F. F. S. 2020, A\&A, 644, L2

Stassun, K. G., Oelkers, R. J., Paegert, M., et al. 2019, AJ, 158, 138

Suissa, G., Wolf, E. T., Kopparapu, R. K., et al. 2020, AJ, 160, 118

Thompson, M. A. 2021, MNRAS, 501, L18

Van Eylen, V., Agentoft, C., Lundkvist, M. S., et al. 2018, MNRAS, 479, 4786

Van Eylen, V., Astudillo-Defru, N., Bonfils, X., et al. 2021, MNRAS, 507,2154

Villanueva, G., Cordiner, M., Irwin, P., et al. 2020, arXiv:2010.14305

Wang, J., Wang, J., Ma, B., et al. 2020, AJ, 160, 150

Wunderlich, F., Godolt, M., Grenfell, J. L., et al. 2019, A\&A, 624, A49

Wunderlich, F., Scheucher, M., Grenfell, J. L., et al. 2021, A\&A, 647, 48 